\title{
Bone and Muscle Crosstalk in Aging
}

\author{
Chen $\mathrm{He}^{1}$, Wenzhen $\mathrm{He}^{1}$, Jing Hou' ${ }^{1}$, Kaixuan Chen ${ }^{1}$, Mei Huang ${ }^{1}$, Mi Yang ${ }^{1}$, \\ Xianghang Luo ${ }^{1,2,3}$ and Changjun Li $i^{1,2,3 *}$
}

\begin{abstract}
${ }^{1}$ Department of Endocrinology, Endocrinology Research Center, Xiangya Hospital of Central South University, Changsha, China, ${ }^{2}$ National Clinical Research Center for Geriatric Disorders, Xiangya Hospital of Central South University, Changsha, China, ${ }^{3}$ Key Laboratory of Organ Injury, Aging and Regenerative Medicine of Hunan Province, Changsha, China
\end{abstract}

\section{OPEN ACCESS}

Edited by:

Rebecca Ann Wingert, University of Notre Dame,

United States

Reviewed by:

Sonia Alonso-Martin,

Biodonostia Health Research Institute

(IIS Biodonostia), Spain

Mark Hamrick,

Augusta University, United States

*Correspondence:

Changjun Li

lichangjun@csu.edu.cn

Specialty section:

This article was submitted to

Molecular Medicine,

a section of the journal

Frontiers in Cell and Developmental

Biology

Received: 21 July 2020

Accepted: 09 November 2020

Published: 10 December 2020

Citation:

He C, He W, Hou J, Chen K, Huang $M$, Yang M, Luo X and Li C (2020) Bone and Muscle Crosstalk

in Aging.

Front. Cell Dev. Biol. 8:585644. doi: 10.3389/fcell.2020.585644
Osteoporosis and sarcopenia are two age-related diseases that affect the quality of life in the elderly. Initially, they were thought to be two independent diseases; however, recently, increasing basic and clinical data suggest that skeletal muscle and bone are both spatially and metabolically connected. The term "osteosarcopenia" is used to define a condition of synergy of low bone mineral density with muscle atrophy and hypofunction. Bone and muscle cells secrete several factors, such as cytokines, myokines, and osteokines, into the circulation to influence the biological and pathological activities in local and distant organs and cells. Recent studies reveal that extracellular vesicles containing microRNAs derived from senescent skeletal muscle and bone cells can also be transported and aid in regulating bone-muscle crosstalk. In this review, we summarize the age-related changes in the secretome and extracellular vesicle-microRNAs secreted by the muscle and bone, and discuss their interactions between muscle and bone cells during aging.

Keywords: bone, muscle, crosstalk, aging, osteosarcopenia

\section{INTRODUCTION}

With increasing aging populations, studies regarding aging have become an emerging field. Aging is the process of tissue deterioration coupled with an enormous economic burden. Osteosarcopenia is an age-related pathological condition characterized by porous and fragile bone and sarcopenia (a disease exhibiting low muscle mass and function), recognized as a "hazardous duet," which induces fragile bones and increased risk of fractures, thus leading to high mortality rates and global financial threat. Binkley and Buehring (2009) first coined Sarco-Osteopenia to diagnose simultaneous bone and muscle weakness, calling for scientists to investigate specific mechanisms of the co-existence of osteoporosis and sarcopenia. Fat infiltration is a common character of aged bone and muscle; besides, osteoporosis and sarcopenia share several genetic, developmental, and endocrine factors. Endocrine factors have become a hot spot for studying the pathogenesis of osteosarcopenia, especially regarding myokines and osteokines.

Myokines, defined as soluble molecules expressed and released by muscle fibers, regulate the biological and pathological activities of local and distant cells and organs. McPherron et al. (1997) identified the first myokine, i.e., myostatin, which is expressed in developing and mature muscle and negatively regulates muscle mass. Steensberg et al. (2000) reported that contracting muscle releases interleukin- 6 into the bloodstream, and realized that muscle could function as an endocrine organ. Moreover, with the development of modern sequencing and analytic technology, about 672 myokines were identified (Grube et al., 2018). They are composed mainly of peptides, such as growth factors, cytokines, and some small organic acids. Various myokines in the circulation 
work together to maintain homeostasis via muscle-organ crosstalk (Das et al., 2020). For example, contracting muscle secretes brain-derived neurotrophic factors to improve the memory and learning capacity, and exercise-induced irisin was reported to promote thermogenesis and increase bone mass (Vaynman et al., 2004; Boström et al., 2012; Colaianni et al., 2015; Severinsen and Pedersen, 2020).

Osteokines, a combination of two Greek words ("osteo" meaning "bone" and "kino" meaning "movement"), represent bone cell-derived factors, which influence local and systemic metabolism. The bone marrow microenvironment is complex and comprises diverse cells. Cells that participate in bone metabolism include bone marrow mesenchymal stem cells (BMSCs), osteoblasts, osteocytes, osteoclasts, and their precursor cells. The most widely studied osteokines are of osteoblast, osteocyte, and osteoclast origin. For instance, receptor activator of nuclear factor kappa B ligand (RANKL) and osteoprotegerin (OPG) from osteoblasts, sclerostin (SOST), fibroblast growth factor 23 (FGF23), and RANKL from osteocytes, and receptor activator of nuclear factor kappa B (RANK) from osteoclasts etc. Unlike skeletal muscle, from which over 600 myokines were identified, bone was not recognized as an endocrine organ until 2007 (Lee et al., 2007). In addition, the research regarding bonederived factors mediating bone-muscle crosstalk is limited.

Besides the cytokines, growth factors and proteases released by aging cells, which are defined as the senescence-associated secretory phenotype (SASP), are harmful to musculoskeletal function. The nuclear acid changes in aging cells reportedly induce dysfunction in their parent cells (Yang et al., 2017; Li et al., 2018; Xiao Y.Z. et al., 2020). Recently, extracellular vesicles holding the above factors shed from senescent cells have also been recognized as another type of SASP (Alibhai et al., 2020).

Extracellular vesicles play an important role in cell-to-cell communication, as they can circulate in blood and dock into distant target cells to exert regulatory roles. Extracellular vesicles (EVs) can be divided into many types depending on their size, synthesis, and secretion mechanisms. Current methods cannot isolate profoundly pure subsets; therefore, we prefer the umbrella term "extracellular vesicles." Two main subtypes of EVs exist, including exosomes, which are "cup shaped" membranous vesicles between 30 and $150 \mathrm{~nm}$ released by late endosome fusion with plasma membranes, and microvesicles, irregularly shaped vesicles measuring 100-1,000 nm, which originate directly from cell membranes. EVs hold microRNAs, proteins, mRNAs, lipids, etc., bind with or are endocytosed by recipient cells, transport their cargo to target cells to modulate biological activities. Recently, many articles describe the regulatory effects of senescent cell-released EVs in various pathologic conditions. Kadota et al. (2018) had reviewed the effects that senescent-cell-EVs exert on age-related lung diseases. In addition, enhanced miR-29b-3p expression in senescentBMSC-EVs accounts for insulin resistance during aging (Su et al., 2019). However, there are few researches investigating the effects of EVs released from aging cells on musculoskeletal homeostasis. For instance, researchers found that raising miR-31 in aged BMSC and endothelial cell EVs suppress bone formation and enhance bone resorption, likewise increased miR-31 could inhibit dystrophin response to loading, and cause muscles of aged rats more susceptible to injury after disuse (Weilner et al., 2016; Hughes et al., 2018; Xu R. et al., 2018).

There are many reviews regarding musculoskeletal communication for osteosarcopenia, but they mainly focus on simple bone-muscle crosstalk; aging and nutrition are included as additional factors to emphasize that dysfunction of skeletal muscle and bone always occur together (Reginster et al., 2016; Li et al., 2019). In this review, we focus on bone-muscle crosstalk during aging, briefly describe several classical and new myokines and osteokines (Tables 1, 2 and Figure 1), discuss how EV-microRNAs change with age (Table 3 and Figure 2), and finally point out the shortcomings of present studies and provide prospects of the potential field in age-related musculoskeletal diseases.

\section{PROTEOME ALTERED WITH AGE IN BONE-MUSCLE CROSSTALK}

\section{Myokine \\ Irisin}

Irisin, a polypeptide comprising 112 amino acids, is a messenger derived from skeletal muscle to regulate metabolism. Boström et al. (2012) revealed that irisin, a proteolytic product with amino terminal residues of fibronectin type III domain containing 5 (FNDC5), acts as an exercise-induced myokine to promote thermogenesis by browning white fat. Besides, Boström et al. (2012) revealed that irisin might function in glucolipid metabolism. Lately, it has been confirmed to rescue obesity in high-fat diet animal models and improve insulin resistance by targeting genes involved in glucose transport and utilization, such as solute carrier family 2, member 4 (Glut4) and phosphoenolpyruvate carboxykinase (Pck) (Xin et al., 2016). In addition, irisin was reported to promote pancreatic $\beta$ cell proliferation and protect it from apoptosis to directly maintain pancreatic function. Exercise increases serum irisin level, furthermore, exercise is of great benefit to bone remodeling. Therefore, irisin was chosen for further investigation to determine whether it is engaged in muscle-bone communication in vitro and in vivo.

\section{The effects of irisin on BMSCs}

Colaianni et al. (2014) reported that irisin is continuously released at a basal level by muscle and enhanced during exercise. BMSCs cultured in myoblast conditioned medium exhibit more alkaline phosphatase (ALP)-positive colonies (Colaianni et al., 2014). Furthermore, they found that recombinant irisin injection in young male mice once a week at a low dose of $100 \mu \mathrm{g} / \mathrm{kg}$ for 4 weeks increases cortical bone mass and stimulates osteoblastic bone formation without altering energy metabolism, indicating that irisin promotes expression of activating transcription factor 4 (Atf4), which subsequently enhances the biological activity of runt-related transcription factor 2 (RUNX2) and a series of osteogenic transcription factors, enabling BMSCs to develop into the osteogenic lineage (Colaianni et al., 2015). In addition, irisin can improve the colony formation ability of BMSCs and enhance 
TABLE 1 | Myokines altered with age in bone-muscle crosstalk.

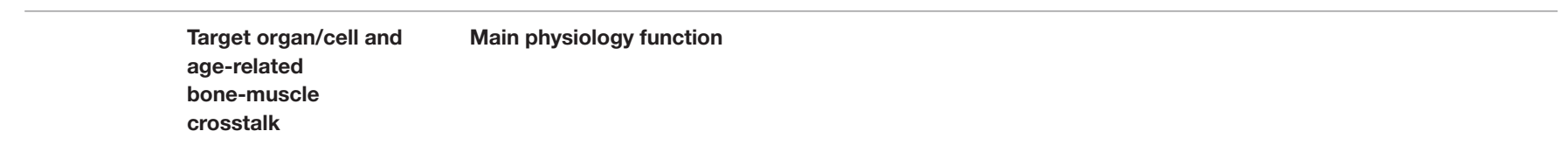

\section{Myokines}

Irisin

$$
\text { BMSCs }
$$

Osteoblasts

Osteocytes

Osteoclasts

Muscle

Age-related

bone-muscle crosstalk

Myostatin BMSCs

Osteoblasts

Osteocytes

Osteoclasts

Muscle

Age-related

bone-muscle crosstalk
Increase ALP positive colonies (Colaianni et al., 2014).

Promote BMSCs develop into osteogenic lineage via ATF4-dependent RUNX2 activation (Colaianni et al., 2015). Rescue colony formation and ALP, Collagen I expression of BMSCs from suspended mice (Colaianni et al., 2017).

Promote osteoblast proliferation and differentiation via MAPK pathway (Colaianni et al., 2015; Qiao et al., 2016).

Reduce osteoblast apoptosis (Xu et al., 2020).

Preserve osteoblast differentiation via $\beta$-Catenin in suspended mice (Chen et al., 2020).

Polarize Macrophages into M2 phenotype via AMPK pathway then promote osteoblast differentiation (Ye et al., 2020).

Assist osteocyte-related osteocytic osteolysis and increase sclerostin expression (Kim et al., 2018).

Downregulate SOST expression (Storlino et al., 2020).

Inhibit the apoptosis of osteocytes under oxidative stress and/or microgravity via decreasing mitochondrial pathway of cell death (Storlino et al., 2020).

Regulate osteoclast formation and activities via modulating OPG expression (Colaianni et al., 2017).

Reduce expression of Cathepsin K, Rank and Nfatc1 mRNA level (Ma et al., 2018).

Promote proliferation and decrease differentiation of osteoclast precursors through p38, JNK and RANKL-induced

NF-кB pathway separately (Ma et al., 2018).

Enhance the mRNA level of muscle growth-related gene like Igf1 and Pgc1 $\alpha 4$ through ERK signaling pathway and suppress the expression of myostatin (Huh et al., 2014).

Improve the proliferation and fusion of myoblasts; enhance protein synthesis via activation of AKT and ERK; activate satellite cells pool; increase expression of II-6, then facilitate myogenesis (Reza et al., 2017b).

Improve sarcolemmal stability or increase phosphorylation of FOXO3 $\alpha$ and attenuate chymotrypsin-like enzyme activity (Reza et al., 2017a; Chang and Kong, 2020).

Promote proliferation of C2C12 cells via increasing chemokine (C-C motif) ligand 7 (Lee et al., 2019).

Negatively correlated with age (Huh et al., 2012; Rana et al., 2014).

Increase with age (Mahmoodnia et al., 2016; Ruan et al., 2019).

Use as a biomarker to predict pre-sarcopenia and sarcopenia (Chang et al., 2017).

Positive association with bone mineral status and low irisin level is related to increased hip fracture risk (Yan et al., 2018).

Promote mesenchymal multipotent cells differentiate toward adipocytes (Hamrick et al., 2007).

Induce the expression of Ppar $\gamma$ and C/EBP $\alpha$ (Rebbapragada et al., 2003).

Decrease the mechanosensitivity of BMSCs (Hamrick et al., 2007).

Inhibit expression of Alp, osteocalcin, osterix and Runx2 (Chen et al., 2017; Zhang et al., 2020).

Decrease the number of osteoblasts on bone surface (Chen et al., 2017).

Low-intensity pulsed ultrasound inhibits myostatin to enhance the proliferation of osteoblasts (Sun et al., 2019).

Increase the expression of Wnt pathway inhibitor SOST, DKK1, besides, promote RANKL expression in osteocytes (Qin et al., 2017).

Reduce the miR-218 expression in osteocytes-derived exosomes and then impairs osteoblastic differentiation via Wnt pathway (Qin et al., 2017).

Increase the expression of Nfatc1 and the number of TRAP + multinucleated giant cells (Chen et al., 2017).

Enhance expression of integrin $\alpha$, integrin $\beta 3$ and calcitonin receptor via enhancing SMAD2-dependent NFATc1 nuclear translocation (Dankbar et al., 2015).

Myostatin-null mice show muscle hypertrophy (Shan et al., 2013).

Myostatin inhibition by GASP-2 can promote proliferation and differentiation of myoblasts (Perie et al., 2016).

Downregulate Pax7 to suppress activation and self-renew of quiescent satellite cells; arrest C2C12 myoblasts in G1

phase through promoting P21 expression; decrease Pax3, Myod1, and Myf5 expression to suppress differentiation

(Rodriguez et al., 2014).

Enhance ribosome biogenesis through activating S6K and rpS6 (Rodriguez et al., 2014).

Decrease protein synthesis via inhibiting eEF2K-eEF2 after AMPK phosphonation (Deng et al., 2017).

Promote the gene expression of Murf-1, enhance the proteasome activity of 26S and increase the expression of autophagy-related genes, such as Atg3, Atg12, etc. (Wang D.T. et al., 2015).

Increase with age until 57 years old, then it exhibits a decrease trend (Szulc et al., 2012).

Myostatin level is periodic, and exhibit the highest concentration in spring (Szulc et al., 2012).

Increase or decrease with age (Bowser et al., 2013; Poggioli et al., 2016).

Myostatin in fast muscle fiber ensued with aging (Shibaguchi et al., 2018).

Mstn deficiency improve muscle atrophy and reduced muscle capacity in aging mice (Siriett et al., 2006).

Myostatin inhibitor can be used to treat age-related sarcopenia (White and LeBrasseur, 2014).

High myostatin level is related to low BMD subjects in Chinese elderly (Wu et al., 2018).

Myostatin inhibition enhance osteogenesis induced by aged myofibers and myoblast cell line (Zhang et al., 2020). 
TABLE 1 | Continued

\begin{tabular}{|c|c|c|}
\hline & $\begin{array}{l}\text { Target organ/cell and } \\
\text { age-related } \\
\text { bone-muscle } \\
\text { crosstalk }\end{array}$ & Main physiology function \\
\hline \multirow[t]{11}{*}{ FGF21 } & BMSCs & $\begin{array}{l}\text { Favor BMSCs commitment into adipocytes over osteoblast lineage via PPAR } \gamma \text {-FGF21 feed-forward regulatory pathway } \\
\text { (Wei et al., 2012). }\end{array}$ \\
\hline & \multirow[t]{3}{*}{ Osteoclasts } & $\begin{array}{l}\text { Bone resorption enhanced in Fgf21 transgenic mice possibly via increasing the expression level of RANKL in osteocytes } \\
\text { (Wei et al., 2012). }\end{array}$ \\
\hline & & $\begin{array}{l}\text { Promote RANKL-induced osteoclastogenesis via promoting IGFBP1 release by liver then combination with Integrin } \beta 1 \\
\text { receptor in osteoclasts (Wang X. et al., 2015). }\end{array}$ \\
\hline & & $\begin{array}{l}\text { MiR-100 overexpression causes reduced FGF21 level thus inhibit osteoclasts-mediated bone resorption and partially } \\
\text { restore the bone phenotype in OVX-operated mice (Zhou et al., 2019). }\end{array}$ \\
\hline & \multirow[t]{4}{*}{ Muscle } & $\begin{array}{l}\text { Dispensable for muscle mass maintenances in normal condition and necessary to induce muscle atrophy and } \\
\text { weakness in starvation state via enhancing BNIP3 activation (Oost et al., 2019). }\end{array}$ \\
\hline & & $\begin{array}{l}\text { Reduction of OPA1 is related to aging-related muscle atrophy, while Fgf21 deletion is reported to partially rescue the } \\
\text { muscle loss caused by OPA1 deficiency (Tezze et al., 2017). }\end{array}$ \\
\hline & & $\begin{array}{l}\text { Induce cell cycle exit in C2C12 myoblasts through suppressing the cell cycle-related protein sequential to activation of } \\
\text { p21 induced by p53 (Liu S. et al., 2017; Liu et al., 2017b. }\end{array}$ \\
\hline & & $\begin{array}{l}\text { Enhance myogenesis via activating PAX3, and promote formation of smaller aerobic myofibers during fasting via the } \\
\text { FGF21-SIRT1-AMPK-PGC1 } \alpha \text { pathway (Liu S. et al., 2017; Liu et al., 2017a,b) }\end{array}$ \\
\hline & Age-related & Increase with age (Hao et al., 2018; Lee et al., 2020). \\
\hline & bone-muscle crosstalk & Negative associated with BMD (Hao et al., 2018; Lee et al., 2020). \\
\hline & & Positive relevant with the sarcopenia (Hanks et al., 2015; Tezze et al., 2017). \\
\hline \multirow[t]{5}{*}{ BAIBA } & Osteoblasts & $\begin{array}{l}\text { Promote proliferation or enhance the expression of Runx2, Opg, osteopontin, and Alp via moderate ROS induced by } \\
\text { NAD(P)H oxidase } 4 \text { (Zhu X.W. et al., 2018). }\end{array}$ \\
\hline & Osteocytes & $\begin{array}{l}\text { Protect young osteocytes from ROS induced cell death via protecting the mitochondrial morphology and function } \\
\text { (Kitase et al., 2018). }\end{array}$ \\
\hline & Muscle & $\begin{array}{l}\text { BAIBA can prevent loss of EDL and soleus muscle function in unloading hindlimb male mice (Jung et al., 2015). } \\
\text { BAIBA prevent hyperlipidemia-induced insulin resistance and palmitate-induced inflammation in C2C12 myoblast cell } \\
\text { line (Kitase et al., 2018). }\end{array}$ \\
\hline & $\begin{array}{l}\text { Age-related } \\
\text { bone-muscle crosstalk }\end{array}$ & $\begin{array}{l}\text { The protective effect of BAIBA on osteocytes decreasing with age due to reduction of BAIBA receptor-MRGPRD in } \\
\text { osteocytes (Kitase et al., 2018). }\end{array}$ \\
\hline & & Aged skeletal muscle produces more BAIBA (Kitase et al., 2018). \\
\hline \multirow[t]{3}{*}{ METRNL } & Osteoblasts & Inhibit mineralized nodule formation (Gong et al., 2016). \\
\hline & Muscle & Wait to explore. \\
\hline & $\begin{array}{l}\text { Age-related } \\
\text { bone-muscle crosstalk }\end{array}$ & Wait to explore. \\
\hline
\end{tabular}

the expression of previously impaired genes, such as $A l p$ and collagen I, in hind-limb suspended mice (Colaianni et al., 2017).

\section{The effects of irisin on osteoblasts}

Increased bone formation not only can be realized by BMSC osteogenic bias but also promoted by enhanced osteoblast proliferation and differentiation. Mitogen-activated protein kinase (MAPK) signaling pathways mainly regulate cell proliferation and differentiation, with no exception regarding its role in osteoblasts. Qiao et al. (2016) illustrated that after treatment with irisin, basal MAPK14 (p38) and total MAPK1 (ERK) remain constant in osteoblasts, but expression levels of phosphorylated p38 and phosphorylated ERK increase and peak 5-20 min after stimulus. Pretreatment of primary rat osteoblasts and MC3T3-E1 cells with p38 inhibitor SB203580, or ERK inhibitor U0126 eliminate the effect of irisin on proliferation and differentiation (Qiao et al., 2016). Consistent with that, Colaianni et al. (2015) reported that irisin enhances ERK phosphorylation in cultured osteoblasts. Furthermore, irisin can reduce the number of apoptotic osteoblasts in an osteoporosis rat model, and protect against decreased osteoblast differentiation by increasing $\beta$-catenin levels (Chen et al., 2020; Xu et al., 2020). Except for its direct effect on osteoblasts, irisin can indirectly promote osteogenesis by polarizing macrophages to the M2 phenotype via the adenosine monophosphate-activated protein kinase (AMPK) pathway (Ye et al., 2020).

\section{The effects of irisin on osteocytes}

Kim et al. (2018) first discovered the irisin receptor, $\alpha \mathrm{V}$ Integrin, in osteocytes. They reported that an osteocyte-related direct bone resorption model, ovariectomy (OVX)-induced Osteocytic osteolysis, requires Fndc5 and sclerostin to induce bone resorption. Moreover, sclerostin, a protein specifically expressed in osteocytes, appears increased in a dose-dependent manner after culture with irisin (Kim et al., 2018). Nevertheless, Colaianni et al. (2015) showed that irisin level is negatively correlated with sclerostin. Kim et al. (2018) hypothesized that irisin plays a bidirectional role depending on its exposure mode, like the parathyroid hormone (PTH). Lately, Storlino et al. (2020) demonstrated that SOST expression is downregulated by intermittent irisin administration, while continuous irisin exposure doesn't modify SOST expression. Moreover, they found 
TABLE 2 | Osteokines altered with age in bone-muscle crosstalk.

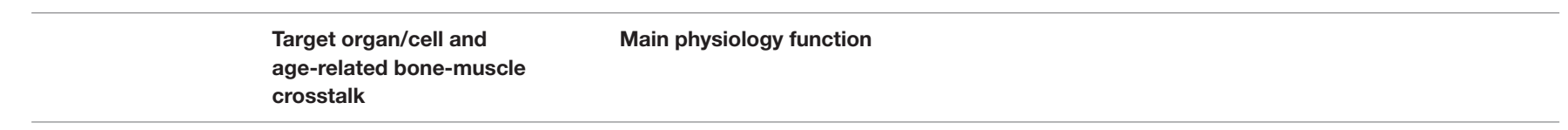

\section{Osteokines}

Osteocalcin

Osteocalcin

Bone

Age-related bone-muscle crosstalk

Sclerostin

Muscle

Bone

Age-related bone-muscle crosstalk

OPG/RANKL/RANK

Muscle

Bone

Age-related bone-muscle crosstalk
Improve the ability of muscle to utilize glucose and fatty acids and form a feed-forward axis to amplify the role in adaption to exercise (Mera et al., 2016a).

Necessary to maintain muscle mass in old mice via promoting protein synthesis (Mera et al., 2016b).

Enhance $\mathrm{C} 2 \mathrm{C} 12$ myoblast cell proliferation and myogenic differentiation thorough activation of the PI3K/Akt, p38 and GPRC6A-ERK1/2 signaling pathway separately (Liu S. et al., 2017). Positive associated with muscle mass or function (Levinger et al., 2014; Harslof et al., 2016; Lin et al., 2016).

Marker of bone formation, but Ocn ${ }^{-/-}$mice exhibit an increased bone mass (Li J. et al., 2016).

Suppress bone formation when coupled to a Gi-protein in human osteoblast cell line (Bodine and Komm, 1999).

Promote bone mineral mature in an Ocn knockout mice and hydroxyapatite crystal growth was suppressed by OCN in certain studies (Neve et al., 2013).

Recruit osteoclasts and promote osteoclastogenesis or enhance its chemotaxis (Neve et al., 2013; Li J. et al., 2016).

Enhanced RANKL-dependent bone resorption mediated by muscle released IL-6 (Mera et al., 2016a).

Decrease with age (Mera et al., 2016a; Diemar et al., 2020).

Negative association with BMD (Kim et al., 2010; Ling et al., 2016).

Maintain muscle mass and improve the muscle phenotype of aged mice (Mera et al., 2016a,b)

Sost ${ }^{-/-}$mice exhibit an increasing trend of lean body mass fraction and overexpress sclerostin by an adeno-associated virus showing a significant reduction in lean body mass (Kim S.P. et al., 2017).

Sclerostin is higher in low muscle mass subjects than healthy controls (Kim et al., 2019).

Suppress Wnt3a mediated crosstalk between the MLO-Y4 osteocytes and muscle cells C2C12 via regulating Wnt/ $\beta$-catenin pathway (Huang et al., 2017).

Sclerostin inhibition restore muscle function in cancer induced muscle weakness (Hesse et al., 2019).

Cannot prevent soleus muscle atrophy in rodents after spinal cord injury, probably because minimally expression of Lrp5/Lrp6 in the rat soleus (Phillips et al., 2018).

Various modifications in or near SOST can cause abnormal bone mineral density such as Sclerosteosis and Van Buchem disease (Holdsworth et al., 2019).

Sclerostin transgenic mice exhibit osteopenia (Winkler et al., 2003).

Regulate differentiation and mineralization of osteogenic lineage cell via a MEPE-ASARM dependent mechanism (Atkins et al., 2011).

Sclerostin inhibition could reactive quiescent bone lining cells (Kim S.W. et al., 2017).

Ensues with aging (Mirza et al., 2010; Ota et al., 2013).

Lower sclerostin level in osteoporosis patients (Reppe et al., 2015).

Ablation of Sost showed an increasing trend of lean body mass in elderly animals (Kim S.P. et al., 2017). A positive relevance between sclerostin and sarcopenia in type 2 diabetes mellitus (Medeiros et al., 2020).

RANKL-RANK interaction activates NF-кB, a pro-inflammation pathway which can inhibit myogenic differentiation and enhance the expression of ubiquitin proteasome system to induce muscle atrophy. Anti-RANKL protect muscle from chronic inflammation and improve its mechanical properties through shifting macrophages into M2 phenotype and inhibit the activation of NF-кB (Hamoudi et al., 2019). Dystrophic mdx mice specifically knockout Rank in muscle rescue its maximum specific force, while full-length OPG-Fc treatment activates sarco/endoplasmic reticulum $\mathrm{Ca}^{2+}$ ATPase to regulate $\mathrm{Ca}^{2+}$ homeostasis in dystrophic EDL through another pathway (Dufresne et al., 2018).

RANKL inhibitor Dmab can rescue muscle function in human (Bonnet et al., 2019).

Opg knockout mice displayed selective atrophy of fast-twitch-type Ilb myofibers atrophy and C2C12 myotube exhibited reduced cross section area when exposure to RANKL (Hamoudi et al., 2020).

RANKL-RANK activate major molecules in downstream pathway signaling, like nuclear factor NF- $\mathrm{B}$ and colony stimulating factor 1 receptor et al., thereby driven pre-osteoclasts to differentiate into osteoclasts (Chung et al., 2014).

Opg ablated mice showed increased adipocytes and BMSCs from these mice prefer to adipogenesis via enhanced expression of adipogenic transcription marker, Ppar $\gamma$ (Zhang et al., 2016). RANK in osteoclasts derived exosomes induce reverse RANKL signaling leading to increased osteogenesis and mineralization (Ikebuchi et al., 2018).

RANKL/OPG ratio, a preferable harmful determinant of bone mass, increase with age (Chung et al., 2014; Zhang et al., 2016; Piemontese et al., 2017).

IL-3 can improve the RANKL/OPG ratio and act as a potent medicine to treat osteoporosis (Singh et al., 2018).

RANKL inhibitor Dmab can rescue muscle function in postmenopausal women, and also in osteo-sarcopenic Pparb-/- mice (Bonnet et al., 2019). 


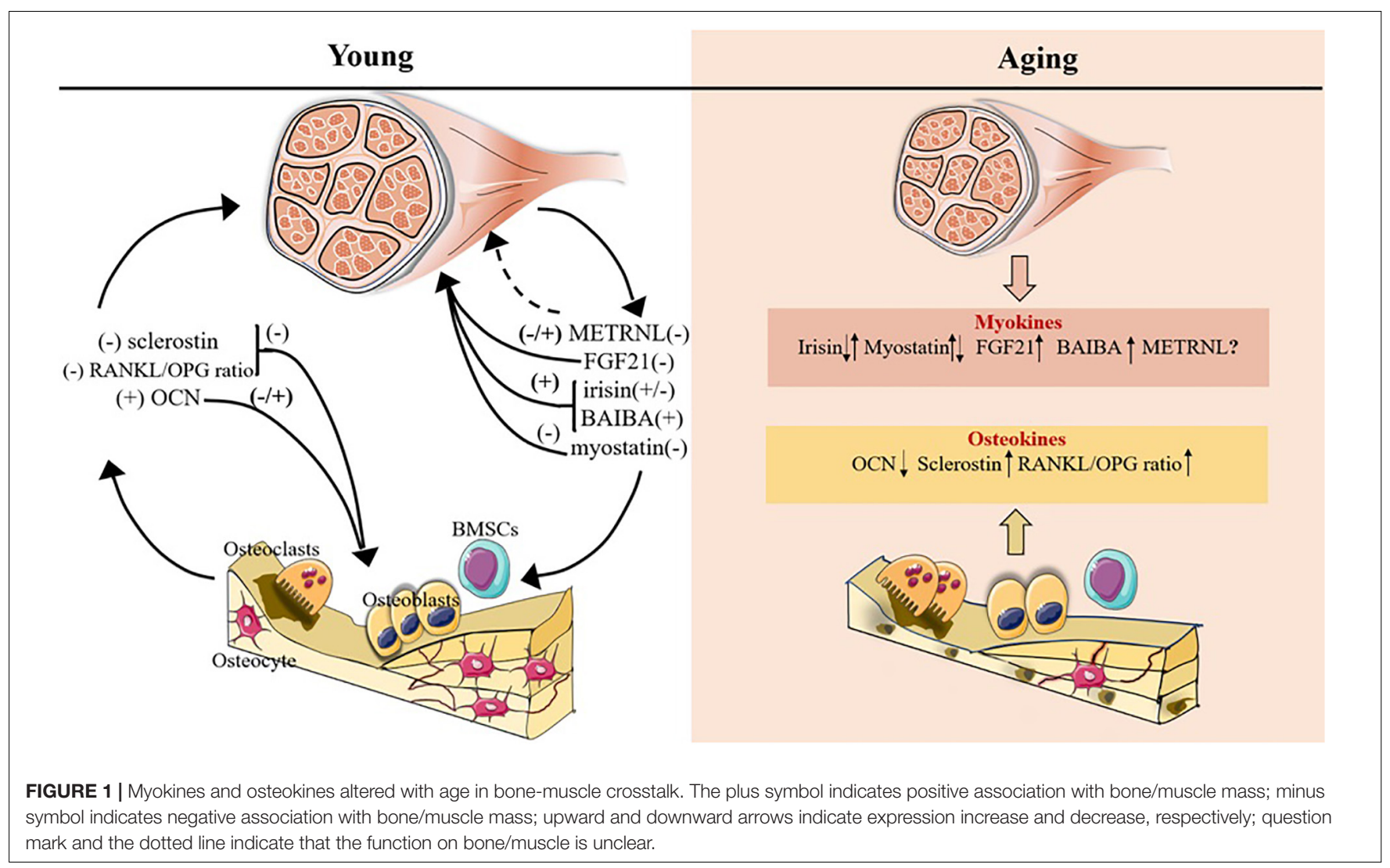

TABLE 3 | Potentially different expression of senescence-EVs-microRNAs in bone-muscle crosstalk.

\begin{tabular}{|c|c|c|}
\hline & $\begin{array}{l}\text { Target organ/cell and } \\
\text { age-related bone-muscle } \\
\text { crosstalk }\end{array}$ & Main physiology function \\
\hline & Muscle & EVs-miR-27 might be an active player, and wait to explore. \\
\hline \multirow[t]{3}{*}{$\begin{array}{l}\text { EVs-microRNAs from } \\
\text { bone cells }\end{array}$} & Bone & $\begin{array}{l}\text { Osteocytes-derived EVs miR-218 can be suppressed by myostatin, then taken up by osteoblasts } \\
\text { inhibiting osteoblastic differentiation (Qin et al., 2017). }\end{array}$ \\
\hline & Muscle & Wait to explore. \\
\hline & $\begin{array}{l}\text { Age-related bone-muscle } \\
\text { crosstalk }\end{array}$ & $\begin{array}{l}\text { Aged BMSCs express high level of miR-31a-5p leading to bias lineage fate of BMSCs to adipocytes } \\
\text { through SATB2, and it release exosomes with high level of miR-31a-5p, then increase the number and } \\
\text { function of osteoclasts via Rho pathway (Xu R. et al., 2018). } \\
\text { Aged osteoclasts were found to liberate EVs unloading enhanced expression of miR-214-3p, increasing } \\
\text { miR-214-3p not only promote osteoclast formation but also embracing in osteoclasts-derived EVs then } \\
\text { lead to impaired bone formation ( } L i \text { D. et al., 2016). } \\
\text { MiR-183-5p increase with age in bone-derived EVs and endocytosed by BMSCs to suppress } \\
\text { osteogenic differentiation and lead to senescence (Davis et al., 2017). } \\
\text { Role of miR-15b, miR-17, miR-183, miR-186, miR-188, miR-20a, miR-214, miR-221, miR-24, miR-31, } \\
\text { miR-328, miR-365, and miR-374, and miR-99b etc. in aged bone cells-EVs in osteosarcopenia wait to } \\
\text { explore. }\end{array}$ \\
\hline
\end{tabular}

that irisin inhibits the apoptosis of osteocytes under oxidative stress and/or microgravity (Storlino et al., 2020). Apoptosis inhibition can be explained by the decreased mitochondrial (intrinsic) pathway of cell death. Specifically, irisin can enhance the expression of mitochondrial transcription factor $\mathrm{A}$ and increase the ratio of $\mathrm{B}$ cell leukemia 2 (BCL2)/BCL2-associated 


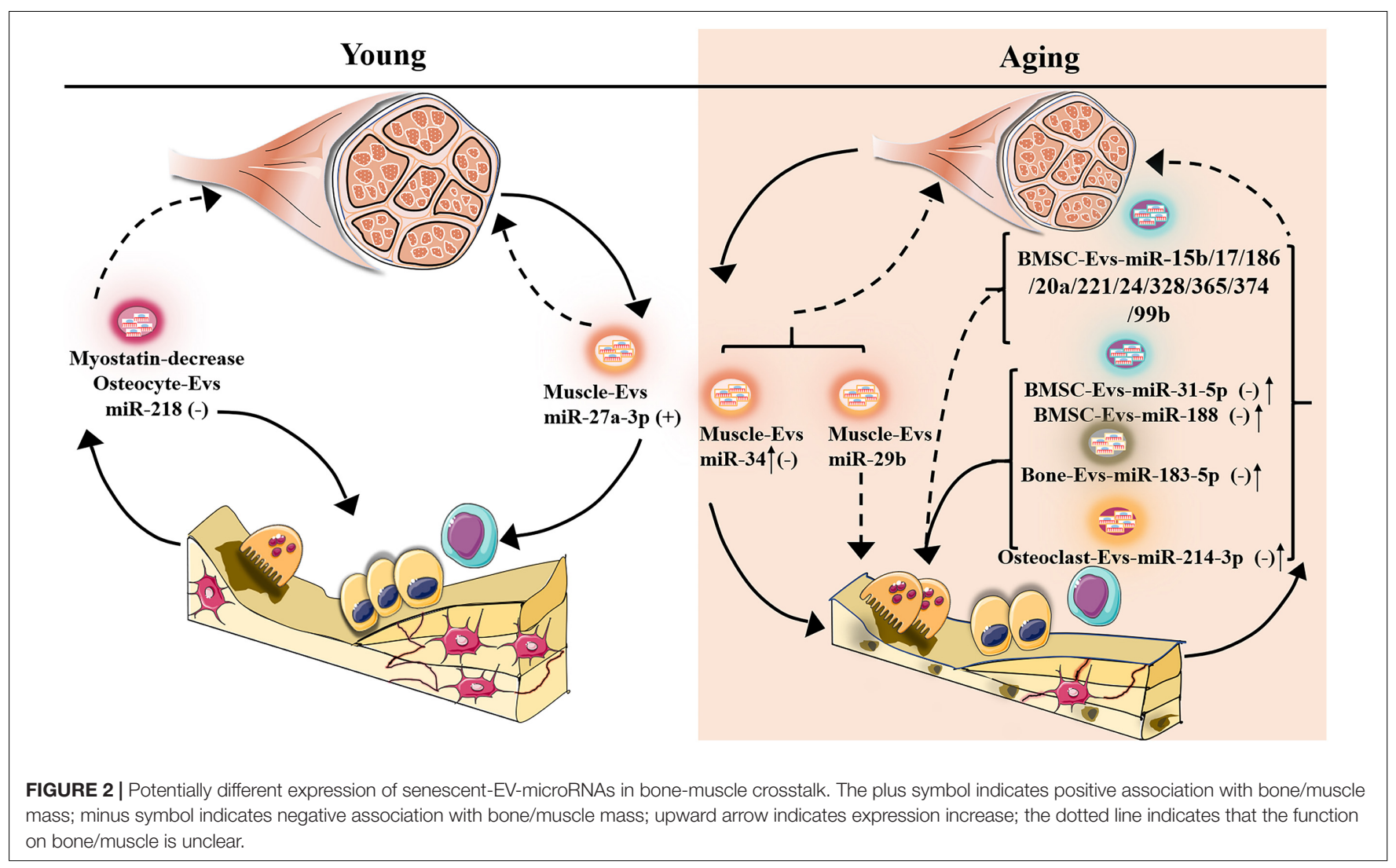

$\mathrm{X}$ protein (BAX), while high BCL2 level can impair BAX initiating autoactivation of caspase- 9 , then block the activation of the executioner, caspase-3. Besides, it can improve the expression of Podoplanin and Connexin 43, genes important for osteocyte dendrite and gap junction channel formation, respectively (Storlino et al., 2020). In addition, irisin may target osteocytes first, then modulates OPG expression, thus decreasing the RANKL/OPG ratio to regulate osteoclast formation and activities (Colaianni et al., 2017).

\section{The effects of irisin on osteoclasts}

Besides the effects on BMSCs and osteoblasts etc., Colaianni et al. (2015) also found that irisin-treated mice exhibit significantly lower number of osteoclasts. Consistent with the above result, irisin was found to reduce the phosphatase acid tartrate resistant (TRAP)-positive multinucleated cells induced by disuse, and it can reduce expression of Cathepsin $\mathrm{K}$, Rank, and nuclear factor of activated T cells cytoplasmic 1 (Nfatcl) mRNA levels significantly (Ma et al., 2018). The detailed mechanism may be explained by the fact that irisin promotes proliferation and decreases differentiation of osteoclast precursors through the p38, c-Jun amino terminal kinase (JNK) signaling pathways and the RANKL-induced nuclear factor kappa B (NF-KB) pathway (Ma et al., 2018). Furthermore, Ibanez et al. (2014) and Xu et al. (2020) found that irisin could upregulate nuclear factor E2related factor 2 (NRF2) expression levels, while Nrf2 knockout increases the survival rate of osteoclasts. This indicates that irisin might decrease the survival of osteoclasts via Nrf2 modification.

\section{The effects of irisin on muscle}

Besides paracrine and endocrine functions, irisin has also been shown to promote myogenesis via autocrine function. Huh et al. (2014) demonstrated that irisin expression increases during myogenic differentiation, furthermore, irisin exposure enhances mRNA levels of muscle growth-related genes, such as insulinlike growth factor 1 (Igfl) and peroxisome proliferative activated receptor gamma coactivator 1 alpha4 (Pgcla4) through the ERK signaling pathway, and suppresses myostatin expression (Huh et al., 2014). An in vivo study conducted by Reza et al. (2017b) showed that irisin injection in normal and pathogenic circumstances cause significant skeletal muscle hypertrophy, accompanied with enhanced grip strength. The underlying mechanisms include (1) irisin improves proliferation and fusion activity of myoblasts; (2) enhances protein synthesis by promoting activation of AKT and ERK; (3) expands the activated satellite cells pool; (4) increases expression of exercise-related genes, such as interleukin-6, thereafter facilitating myogenesis (Reza et al., 2017b). In addition, irisin can partially restore muscle atrophy caused by various etiologies by improving sarcolemmal stability or increasing phosphorylation of forkhead box $\mathrm{O} 3$ alpha $(\mathrm{FOXO} 3 \alpha)$ and attenuating chymotrypsin-like enzyme activity (Reza et al., 2017a; Chang and Kong, 2020). Moreover, irisin administration can enhance $\mathrm{C} 2 \mathrm{C} 12$ myoblasts expressing FNDC5, suggesting that irisin might amplify its positive effect on myogenesis through a regeneration feedback loop (Colaianni et al., 2015). Furthermore, irisin promote $\mathrm{C} 2 \mathrm{C} 12$ proliferation via increased 
chemokine (C-C motif) ligand 7 of active downstream ERK pathway (Lee et al., 2019). However, further investigation is required regarding irisin receptors in muscle cells to understand the mechanisms behind the activation of different signaling pathways.

\section{Irisin in bone-muscle crosstalk during aging}

During aging, decreased mobility and loss of muscle may reduce irisin expression. This hypothesis laid a foundation for follow-up studies investigating the relationship between irisin and aging together with age-related musculoskeletal diseases. The relationship between irisin and age is controversial, with irrelevant, positive, and negative correlation. Huh et al. (2012) reported that irisin level is negatively correlated with age, and it may be induced by age-related muscle loss. Consistent with these results, multiple regression analysis models using backward elimination showed that telomere length is inversely proportional to age and positively associated with plasma irisin level. Telomere length can be predicted by plasma irisin level, and this suggested that age may be negatively associated with irisin level (Rana et al., 2014). Likewise, many epidemiological studies refer to circulating irisin levels growing with age (Mahmoodnia et al., 2016; Ruan et al., 2019). The paradoxical results may be due to researchers using different ELISA kits, which produce varying results, or the inclusion and exclusion criteria set by the researchers differ. More attention should be paid to confirm the genuine relationship between these parameters. In addition, irisin level is positively correlated to human biceps circumference, and irisin was confirmed to use as a biomarker to predict pre-sarcopenia and sarcopenia (Huh et al., 2012; Chang et al., 2017). Furthermore, irisin level is positively associated with bone mineral status, while low irisin level is related to increased hip fracture risk, further corroborating that age-related decreases in muscle and bone may be explained by decreased irisin level, but the detailed mechanism of action remains to be elucidated (Yan et al., 2018).

\section{Myostatin}

Myostatin, also known as growth differentiation factor 8 (GDF8), is a transforming growth factor-beta (TGF $\beta$ ) superfamily protein mainly secreted from muscle fibers. Myostatin is released in an inactive form, then undergoes a series of proteolytic cleavage by bone morphogenetic protein 1/tolloid family of metalloproteinases, and finally produces a mature polypeptide. The hydrolysates $\mathrm{N}$-terminal prodomain can interact with the mature homodimer, counteracting myostatin binding to activin type IIB receptor and activin-like kinase 4 (ALK4) or activinlike kinase 5 (ALK5) heterodimer. Furthermore, follistatin can also combine with myostatin and competitively inhibit myostatin binding to the receptor (Rebbapragada et al., 2003; White and LeBrasseur, 2014). Myostatin is a negative regulator of muscle and strength, and genetic mutation or ablation of the myostatin $(M s t n)$ leads to muscle hypertrophy, enhanced strength, and improved bone architecture (Dankbar et al., 2015). The elaborate biological functions of myostatin in the musculoskeletal system are described below.

\section{The effects of myostatin on BMSCs}

The "colony switch" refers to the fact that once bone marrow stromal cells favor osteogenic differentiation, adipogenesis will be adversely affected. Hamrick et al. (2007) illustrated that BMSCs cultured within an osteogenic medium (obtained from Mstn knockout mice) exhibit more pronounced ALP and alizarin red staining than WT controls, and myostatin exposure induces mesenchymal multipotent cells differentiation toward adipocytes. Consistent with that, Rebbapragada et al. (2003) demonstrated that myostatin induces the expression of adipogenic transcription factors peroxisome proliferatoractivated receptor gamma (Ppary) and CCAAT enhancer binding protein alpha $(C / E B P \alpha)$. Although myostatin ablation promotes osteogenic bias, it cannot restore unloading-induced bone catabolism, which may be due to myostatin-reduced osteogenic differentiation via decreased mechanosensitivity of BMSCs (Hamrick et al., 2007).

\section{The effects of myostatin on osteoblasts}

Unsurprisingly, in vitro exogenous GDF8 can inhibit the expression of Alp, osteocalcin, and two important osteogenic transcription factors, osterix and Runx2, while alizarin red staining is also restrained. Moreover, in vivo, recombinant myostatin decreases the number of osteoblasts on the bone surface, while its neutralizing antibody reverses this effect (Chen et al., 2017). Myostatin inhibition can rescue the ALP signal and RUNX2 expression in aged primary myofiber- and $\mathrm{C} 2 \mathrm{C} 12$ cell line-treated MC3T3-E1 (Zhang et al., 2020). This occurs because the myostatin inhibitor combines with myostatin to reduce negative effects from aged muscle on osteogenic differentiation. Recently, Sun et al. (2019) find that low-intensity pulsed ultrasound could improve bone healing by regulating the myostatin signaling pathway, they deduced that low-intensity pulsed ultrasound promote osteoblast proliferation via inhibiting Smad3-induced $\beta$-catenin stabilization through compromised myostatin signaling pathway.

\section{The effects of myostatin on osteocytes}

Research has reported that myostatin directly affects osteocytes. Osteocytes, comprising $90 \%$ of total bone cells, are deeply embedded in the bone matrix, thus the research on primary osteocytes is limited. Qin et al. (2017) used Osteocytic (Ocy454) cells as an osteocyte-study model, and revealed that myostatin could increase the expression of Wnt pathway inhibitor SOST, dickkopf Wnt signaling pathway inhibitor 1 (DKK1), while promoting the osteoclastogenesis gene Rankl in osteocytes. Moreover, myostatin can reduce miR-218 expression in osteocyte-derived EVs and its parent cells, while miR218 was reported to promote the Wnt pathway by inhibiting SOST expression. Specifically, EVs released by myostatin-treated Ocy454 cells can be taken up by osteoblasts and osteoclast precursor cells during cultured within a medium, and then it can impair osteoblastic differentiation via the Wnt pathway, but exert no effect on osteoclastic differentiation (Qin et al., 2017). This conclusion was made based on in vitro results, and in vivo study is required further. 


\section{The effects of myostatin on osteoclasts}

Besides affecting the expression of RANKL in osteocytes, myostatin can also directly increase the expression of osteoclast differentiation-related genes. Myostatin incubation with RANKL and macrophage colony stimulating factor (M-CSF) treated macrophages increases the number of $\mathrm{TRAP}^{+}$multinucleated giant cells, while it also increases the expression of NFATc1 in a dose-dependent manner (Chen et al., 2017). Likewise, bone surface osteoclasts appeared reduced in myostatin antibodyadministrated mice (Chen et al., 2017). Recently, Dankbar et al. (2015) demonstrate that Mstn knockout mice display lower osteoclast number and smaller osteoclast size, but it seems like myostatin exerts no effect on osteoclast function per se, they also report that myostatin enhances the expression of genes engaged in RANKL-induced osteoclastogenesis, such as Integrin $\alpha$, Integrin $\beta 3$, and calcitonin receptor, via enhancing SMAD2dependent NFATc1 nuclear translocation, which is independent from the ERK pathway.

\section{The effects of myostatin on muscle}

Shan et al. (2013) revealed that myostatin-null mice show muscle hypertrophy. Inhibiting the myostatin canonical pathway by growth and differentiation factor-associated serum protein 2 (GASP-2) was shown to promote proliferation and differentiation of myoblasts (Perie et al., 2016). Regulation of myostatin in myogenesis is profoundly complicated. Rodriguez et al. (2014) elaborated on the mechanism via two schematic illustrations. First, myostatin downregulates paired box 7 (Pax7) to suppress activation and self-renewal of quiescent satellite cells. Second, $\mathrm{C} 2 \mathrm{C} 12$ myoblasts are arrested in the G1 phase through promotion of p21 expression and inhibition of phosphorylated cyclin dependent kinases 2 (CDK2) and cyclin dependent kinases 4 (CDK4). Third, myostatin suppresses differentiation by reducing myogenic genes, such as Pax3, myogenic differentiation 1 (Myod1), and myogenic factor 5 (Myf5), through downregulation of the MEK/ERK1/2 MAPK pathway and/or the AKT/mTORC1 signaling pathway (Rodriguez et al., 2014). Besides affecting the number of muscle fibers, myostatin also decreases its cytoplasmic volume by disrupting the balance between protein synthesis and degradation. Specifically, besides sequential activation of transcription factors eukaryotic translation initiation factor $4 \mathrm{E}$ (eIF4E) and eukaryotic translation initiation factor $4 \mathrm{E}$ binding protein 1 (4E-BP1) in the PI3K/Akt/mTOR pathway, myostatin inhibition was found to affect translation efficiency and ability by enhancing ribosome biogenesis via the activation of ribosomal protein S6 kinase (S6K) and ribosomal protein S6 (rpS6) (Rodriguez et al., 2014). In addition, myostatin can decrease protein synthesis through inhibition of eukaryotic translation elongation factor 2 kinase (eEF2K)-eukaryotic translation elongation factor 2 (Eef2) after AMPK phosphonation (Deng et al., 2017). As for protein degradation, it was reported that myostatin not only promotes gene expression in ubiquitin proteasome, such as tripartite motif-containing 63 (Murf-1), and enhances the proteasome activity of 26S; but also increases the expression of autophagy-related 3 (Atg3), Atg12, etc., which are autophagy-related genes (Wang D.T. et al., 2015).

\section{Myostatin in bone-muscle crosstalk during aging}

Shibaguchi et al. (2018) showed that myostatin increases with age in fast muscle fibers, while myostatin and its receptors in slow muscle are less responsive. This may explain why fast muscle fiber atrophy is characteristic of aging muscle. Opposing results may be explained by species differences (Bowser et al., 2013). Moreover, muscle atrophy and reduced muscle capacity are improved in aging Mstn-deficient mice compared to WT littermates (Siriett et al., 2006). Further, evidence indicates that the myostatin inhibitor can treat age-related sarcopenia (White and LeBrasseur, 2014). Nevertheless, the relevance between age and myostatin is ambiguous. Different opinions exist, with some reporting that circulating myostatin level increases with age until 57 years old, and decreases thereafter, while others implied that serum myostatin increases or decreases with age continuously (Szulc et al., 2012; Bowser et al., 2013; Poggioli et al., 2016). Interestingly, Szulc et al. (2012) found that myostatin level exhibits circannual variation, with greater concentrations observed during spring. These various opinions may due to a highly homologous amino acid sequence-growth and differentiation factor 11 (GDF11), to date no effective method can distinguish GDF11 from GDF8, furthermore, many studies examined circulating total myostatin, including the inactive and active forms, but only biologically active forms are functional. Circulating myostatin levels do not reflect the actual amount in special tissues (Szulc et al., 2012; Shibaguchi et al., 2018; Wu et al., 2018). Moreover, western blot analysis may not reflect true levels of myostatin, as other proteins may appear at the same molecular weight (Poggioli et al., 2016). Regarding the relationship between altered myostatin level and bone status, a cross-sectional study illustrated that high mature myostatin level is significantly related to low bone mineral density (BMD) in elderly Chinese subjects (Wu et al., 2018). Meanwhile, aged myofibers and myoblast cell line enhance osteogenesis upon exposure to myostatin propeptides, which indicates that myostatin may be a potential target to treat osteosarcopenia. Initially, the safety of the myostatin inhibitor was controversial, but later Morissette et al. (2009) dispelled fears that using myostatin inhibitor could cause heart enlargement and showed that it improves insulin sensitivity and bone density (Zhang et al., 2020). In addition, Ran et al. (2020) revealed that anchoring the propeptide to the second extracellular loop of the exosome surface marker CD63 could assist the impaired administration efficacy due to low biological activity and stability. Further research is required to promote myostatin inhibitor as a promising prospect for patients with osteosarcopenia.

\section{Other Myokines}

Other myokines, such as fibroblast growth factor 21 (FGF21), $\beta$ aminoisobutyric acid (BAIBA), and Meteorin-like (METRNL), have been discovered in recent years and reported to affect osteogenesis, myogenesis, and bone-muscle crosstalk during aging. Here, we briefly describe their roles in bone and muscle, and their effects on age-related crosstalk.

\section{Fibroblast growth factor 21}

Fibroblast growth factor 21, abbreviated as FGF21, is weakly expresses in healthy muscle, and it increases with age, starvation, 
and mitochondrial dysfunction (Oost et al., 2019). FGF21 belongs to the FGF family, scientists have found 23 FGF proteins to date, most FGF family proteins regulate cell proliferation, while FGF21 mainly affects metabolism (Itoh, 2014). Despite expressed in muscle, it is also referred to as a hepatokine and adipokine, as the primary organ releasing FGF21 depends on different stimuli (Itoh, 2014). The function of FGF21 on metabolism is controversial, as scientists found that $F g f 21$ knockout prevents high-fat ketogenic diet-induced insulin resistance in mice, but others confirmed the benefits of FGF21 on glucolipid metabolism (Itoh, 2014). Also, the relationship between FGF21 and bone and muscle is intriguing.

The effects of FGF21 on osteogenesis. Wei et al. (2012) firstly identified FGF21 as a skeletal metabolism regulator in 2012, they reported that either FGF21 pharmacological exposure or genetic gain-of-function, causes significantly low bone mass phenotype, moreover, PPAR $\gamma$-FGF21 forms a feed-forward regulatory pathway, which favors BMSCs commitment to adipocytes instead of osteoblast lineage. In addition, they found that osteoclastmediated bone resorption increases in Fgf21 transgenic mice and suggested that FGF21 may promote osteoclastogenesis by increasing RANKL expression level in osteocytes (Wei et al., 2012). Charoenphandhu et al. (2017) and Hao et al. (2018) sequentially confirmed the deterioration effect of FGF21 on skeletal and the indirect influence of FGF21 on osteoclasts in humans and rats. Wang X. et al. (2015) discovered that FGF21 mediates a liver-osteoclast communication via insulinlike growth factor binding protein 1 (IGFBP1). FGF21 enhances the expression of IGFBP1 mainly in the liver, and then IGFBP1 is released into the bloodstream, promotes RANKLinduced osteoclastogenesis via combination with Integrin $\beta 1$ receptor, further activates RANKL-induced NFATc1 expression (Wang X. et al., 2015). Furthermore, miR-100 was identified as the upstream regulator of $F g f 21$, miR-100 overexpression reduces FGF21 levels in serum and liver, thus inhibiting osteoclast-mediated bone resorption and partially restoring the bone phenotype in OVX-operated mice (Zhou et al., 2019). Nevertheless, none of these studies investigated the direct effects of muscle-derived FGF21 on bone and bone-muscle crosstalk.

The effects of FGF21 on myogenesis. Recently, attention is turned to the autocrine function of myokine FGF21. Evidence showed that FGF21 requires other factors to trigger its harmful function in muscle. Oost et al. (2019) demonstrated that Fgf21 is dispensable for muscle mass maintenance under normal conditions, while it is necessary to induce muscle atrophy and weakness in a starvation state via enhancing mitophagic flux mediated by activation of mitophagy protein BCL2/adenovirus E1B interacting protein 3 (BNIP3). Reduction of the important pro-fusion protein optic atrophy protein 1 (OPA1) is related to aging-related muscle atrophy, Tezze et al. (2017) found that in double muscle-specific Opa1/Fgf21 knockout mice, Fgf21 deletion could partially rescue the muscle loss caused by OPA1 deficiency. These observations suggested a harmful effect of FGF21 on muscle. Nevertheless, FGF21 administration cannot inhibit the FGF19 function, which induces muscle hypertrophy (Benoit et al., 2017). Furthermore, FGF21 was found to induce cell cycle exit in $\mathrm{C} 2 \mathrm{C} 12$ myoblasts by suppressing the cell cycle-related protein by $\mathrm{p} 21$, which is activated by p53 (Liu S. et al., 2017; Liu et al., 2017b). In addition, Liu et al. (2017a) suggested that FGF21 enhances myogenesis through PAX3 activation, and it could promote formation of aerobic myofibers via the FGF21-sirtuin-AMPK-peroxisome proliferative activated receptor, gamma coactivator 1 alpha $(\mathrm{PGCl} \alpha)$ pathway in vitro and in vivo. The effect of FGF21 on myogenesis is obscure, and scientists should focus on deciphering these effects to discover novel therapeutic intervention strategies aimed at treating musculoskeletal diseases.

FGF21 in bone-muscle crosstalk during aging. FGF21 was found to increase with age, and reported to be negatively associated with BMD (Hao et al., 2018; Lee et al., 2020). The mechanism behind this may be attributed to BMSCs preferring to form adipocytes over osteoblasts or osteoclasts enhancing its bone resorption via FGF21-IGFBP1 axis (Wei et al., 2012; Wang X. et al., 2015); however, Lee et al. (2020) rejected the second half of the hypothesis and demonstrated that age-related bone loss caused by FGF21 is irrelevant to the FGF21-IGFBP1 axis. Further, serum FGF21 was reportedly positively associated with sarcopenia (Hanks et al., 2015; Tezze et al., 2017). The major shortcoming of this research regarding the relationship between FGF21 with BMD and muscle mass is the cross-sectional study design, as it cannot conclude a causal relationship between agerelated changes in FGF21 and bone or muscle loss in the elderly. Therefore, further research is required to investigate whether FGF21 can be used as a potential target to treat osteosarcopenia, and the causal correlation should be explored.

\section{$\beta$-aminoisobutyric acid}

BAIBA refers to $\beta$-aminoisobutyric acid, which has two enantiomers, L-BAIBA and D-BAIBA, like every other amino acid except for glycine. BAIBA was found in urine and reported as a myokine generated during exercise or from muscle-PGC$1 \alpha$-over-expressing mice. It is a catalysate of branched chain amino acid valine and thymine, exhibiting effects on browning white adipose tissue and promoting $\beta$-oxidation of fatty acid in liver (Roberts et al., 2014). Reports have shown that BAIBA improves metabolism dependent on PPAR and leptin (Kammoun and Febbraio, 2014; Roberts et al., 2014). As a newly discovered exercise-induced myokine, it has attracted many scientists to study its mysterious role in bone-muscle crosstalk.

The effects of BAIBA on osteogenesis. Kitase et al. (2018) found that BAIBA, especially L-BAIBA, could protect young osteocytes from reactive oxygen species (ROS)-induced cell death by protecting mitochondrial morphology and function, and reduce bone loss in a hind-limb unloading model. Subsequently, Zhu X.W. et al. (2018) demonstrated that appropriate low doses of BAIBA either promotes proliferation or enhances the expression of osteogenic transcription factor RUNX2 and differentiation markers OPG, osteopontin, and ALP in MC3T3E1 osteoprogenitor cells. Meanwhile, they declared that moderate ROS production by $\mathrm{NAD}(\mathrm{P}) \mathrm{H}$ oxidase 4 might explain the BAIBA stimulatory effect on an osteoblast cell line (Zhu X.W. et al., 2018). Recently, Wang et al. (2020) illustrate that BAIBA is 
positively associated with BMD in young, healthy women. The correlation is disrupted after the occurrence of a fracture or during an obese state, mainly due to interference caused by medical treatments and cytokines released by obese subjects. Research regarding the effects of BAIBA on bone is far from enough, whether it can affect formation or function of osteoclasts remains to be studied, and its physiological dosages for bone formation also need to be determined.

The effects of BAIBA on myogenesis. Very few BAIBA functional studies on muscle exist. Scientists found that BAIBA could prevent loss of extensor digitorum longus (EDL) and soleus muscle function in unloading hind-limb male mice (Kitase et al., 2018). While BAIBA increases the male EDL muscle mass only, it indicated that gains in muscle mass are unnecessary for improved muscle function and gender specific hormone expression of female mice might account for unaffected muscle phenotype (Kitase et al., 2018). Furthermore, the only in vitro study on the effects of BAIBA on muscle reported that BAIBA prevents hyperlipidemia-induced insulin resistance and palmitate-induced inflammation in a $\mathrm{C} 2 \mathrm{C} 12$ myoblast cell line (Jung et al., 2015). Although it was widely recognized that insulin resistance and muscle inflammation are two mainly harmful factors to muscle mass and function, further research is required to investigate the direct effect of BAIBA on myogenesis (Cleasby et al., 2016).

BAIBA in bone-muscle crosstalk during aging. Reactive oxygen species-induced osteocyte cell death could be rescued by BAIBA treatment. To our knowledge, increased ROS can lead to aging, therefore BAIBA, which protects cells from ROS injury, may be a profoundly effective osteoporosis treatment (Hamrick and McGee-Lawrence, 2018). The protective effect of BAIBA on osteocytes decreases with age; to further elucidate the underlying mechanism, researchers compared not only the ability of skeletal muscle to produce BAIBA in different-aged mice but also the expression of BAIBA receptor-Mas-related G-protein-coupled receptor type $\mathrm{D}$ (MRGPRD) in young and aged osteocytes (Kitase et al., 2018). Interestingly, Kitase et al. (2018) demonstrated that decreasing level of MRGPRD in osteocyte membranes could account for the compromised effect of BAIBA on aged osteocytes, although BAIBA production increases in aged skeletal muscle. Further investigations should be conducted to explore methods to improve MRGPRD expression in osteocytes. Further, the detailed role of BAIBA in osteosarcopenia deserves exploration.

\section{Meteorin-like}

Meteorin-like is a small protein molecule reported to regulate glucose metabolism, thermogenesis, and inflammation (Ushach et al., 2018). Scientists using Pgc-1 $\alpha$ splice isoform Pgc-1 $\alpha 4$ muscle-specific transgenic mice reported that METRNL is released by muscle after exercise; in addition, they found adipocytes can also release METRNL during cold stimulation (Rao et al., 2014). METRNL recruits eosinophils to secret IL4/IL-13, activating macrophages to release anti-inflammatory cytokines to improve glucose homeostasis and promote white adipose tissue (WAT) browning (Rao et al., 2014). In addition, METRNL, also defined as Subfatin, appears downregulated in adipose tissue of calorie restricted rats and is highly expressed in obese-mice adipocytes and during adipogenic differentiation, thus thought to be an adipokine related to energy storage (Li et al., 2014). Moreover, METRNL was found to be an anti-inflammatory cytokine expressed in human barrier tissue and macrophages, while Metrnl knockout mice do not exhibit abnormal metabolism but exhibit immunity defects (Ushach et al., 2018). The reason for this phenomenon may be that, like FGF21, METRNL is weakly expressed under normal conditions and mainly plays roles in regulating immunity, it requires other factors to trigger its function regarding energy metabolism. Furthermore, research investigating the roles of METRNL in bone and muscle regulation is not well-developed, with only one study reporting that METRNL could affect Ap-1 transcription factor and inhibit mineralized nodule formation (Gong et al., 2016). No studies exist regarding the effects of METRNL on skeletal muscle mass and function, but research has shown that METRNL recruits eosinophils to activate macrophages, and eosinophil-macrophage synergy reportedly participates in repairing damaged muscle (Heredia et al., 2013; Rao et al., 2014). Therefore, METRNL supposedly affects muscle regeneration and/or myogenesis. This indicates that METRNL may function as a messenger to mediate muscle-bone crosstalk and their interplay during aging.

\section{Osteokines Osteocalcin}

Osteocalcin (OCN), a secreted protein originating from osteoblasts, goes through sequential cleavages to split amino acid sequences, such as signal peptides, and obtains $\gamma$-carboxylate at three residues aided by a cofactor - vitamin $\mathrm{K}$. There are two forms of OCN depending on the degree of carboxylation, while the under-carboxylated form is considered to function in blood circulation, which stems from an acid circumstance caused by bone matrix resorption through osteoclasts or directly released by osteoblasts during acute stress response (Battafarano et al., 2020). OCN is like hormones in biological characteristic and physiological function. Serum OCN can enter distant cells to regulate insulin resistance, glucose homeostasis, male fertility, and brain function (Li J. et al., 2016; Mizokami et al., 2017; Battafarano et al., 2020). Recently, many studies show that OCN acts as a messenger and participates in interactions between bone and muscle. OCN expression increases dramatically during bone matrix mineralization, which is considered to be a marker representing late osteogenesis. Besides, skeletal muscle, a locomotive and glycogen storage organ, is also an important target tissue of OCN.

\section{The effects of osteocalcin on muscle}

Initially, studies investigating OCN effects on skeletal muscle were limited to energy metabolism. Mera et al. (2016a) demonstrated that OCN could improve muscle to utilize glucose and fatty acids, meanwhile, they found that mice deficient in Ocn exhibit impaired exercise capacity and exogenous OCN administration could rescue the exercise capacity of aged mice. Furthermore, OCN could stimulate skeletal muscle to release IL-6, simultaneously IL-6 and OCN form a feed-forward axis 
to amplify adaption to exercise (Mera et al., 2016a). Next, the influence of OCN on muscle mass was investigated. Using female mice in a study, to exclude the effect of testosterone, Mera et al. (2016b) found that osteocalcin signaling is necessary to maintain muscle mass in old mice by promoting protein synthesis. However, this research did not elaborate on the mechanism. Later, Liu S. et al. (2017) illustrated and confirmed that under-carboxylated OCN enhances $\mathrm{C} 2 \mathrm{C} 12$ myoblast cell proliferation and myogenic differentiation thorough activation of the PI3K/Akt, p38, and GPRC6A-ERK1/2 signaling pathways, respectively. Moreover, several investigations on rats, healthy volunteers, or patients with hypoparathyroidism revealed that under-carboxylated OCN is positively associated with muscle mass or function (Levinger et al., 2014; Harslof et al., 2016; Lin et al., 2016). However, this experiment should be repeated in a large healthy population.

\section{The effects of osteocalcin on bone}

The effects of OCN on bone are divided into three parts. First, the controversial role in bone formation. OCN is highly expressed in mature osteoblasts and a familiar marker of bone formation, however, unexpectedly, $\mathrm{Ocn}^{-1-}$ mice exhibit increased bone mass (Li J. et al., 2016). Bodine and Komm (1999) found an OCN receptor coupled with a Gi-protein in a human osteoblast cell line and confirmed that OCN could suppress bone formation. However, the specific mechanism has not been clarified. Second, the role in bone mineralization. OCN has a strong affinity for $\mathrm{Ca}^{2+}$ and hydroxyapatite and reportedly promotes bone mineral maturity in Ocn knockout mice; furthermore, hydroxyapatite crystal growth is suppressed by OCN in certain studies (Neve et al., 2013). Third, the role in bone resorption, besides recruiting osteoclasts and enhancing its chemotaxis, OCN fragments can also promote osteoclastogenesis (Neve et al., 2013; Li J. et al., 2016). Mera et al. (2016a) further demonstrated that OCN could stimulate skeletal muscle release IL-6, subsequently increasing the expression of RANKL in osteoblasts and decreasing the decoy receptor of Rankl, in other words, OCN enhances bone resorption mediated by IL-6 released from muscle. The detailed mechanism should be elucidated, and researchers should distinguish the functional component between the fragments or carboxylation status.

\section{Osteocalcin in bone-muscle crosstalk during aging}

It is widely recognized that sex hormones and brain function decline with age. Studies reported positive association between serum OCN and testosterone, meanwhile low OCN is related to impaired brain cognition and microstructure (Puig et al., 2016; Zhong et al., 2016). Therefore, although the results regarding OCN and age are controversial, we prefer the theory suggesting plasma OCN levels decrease with age (Mera et al., 2016a; Diemar et al., 2020). Nevertheless, many cross-sectional investigations revealed that OCN is negatively associated with BMD (Kim et al., 2010; Ling et al., 2016). The effects of OCN may be blurred by diverse subtypes, which may explain the contradictory results between age-related bone loss and age-related decreases in OCN, and its deleterious function on bone. Thus, further study is required to confirm whether OCN can be used as a treatment for osteoporosis and sarcopenia.

\section{Sclerostin}

Sclerostin is a glycoprotein released by osteocytes and recognized as a member of Dan/Cerberus family of bone morphogenetic protein (BMP) antagonists. Sclerostin primarily originates from mature osteocytes, but is also observed in the cardio-cerebral vascular system, lung, and kidney, with only the mRNA expression detected in the latter tissues (Delgado-Calle et al., 2017; Holdsworth et al., 2019). Sclerostin not only couples with BMP to suppress BMP-induced phosphorylation of Smad but also impairs the canonical Wnt pathway through competitive combination with LDL receptor related protein 5/6 (LRP5/6), the co-receptor of the Wnt pathway (Winkler et al., 2003; Fijalkowski et al., 2016). In addition, the binding of sclerostin to LRP5/6 requires the "molecular chaperone" LRP4; consequently, mutations in sclerostin or Lrp4 produce similar abnormal phenotype (Fijalkowski et al., 2016).

\section{The effects of sclerostin on muscle}

Sclerostin may play an important role in muscle mass modulation. von Maltzahn et al. (2012) reported that the Wnt signaling pathway is engaged in the differentiation of muscle stem cells. Moreover, Kim S.P. et al. (2017) showed that Sost ${ }^{-/}$mice exhibit increased lean body mass in older animals with $P$-value of 0.06 , while over-expressing sclerostin by an adeno-associated virus revealed the opposite body composition phenotype in Sost ${ }^{-/}$mice, which showed an increase in body fat mass and a small, but significant reduction in lean body mass. Further, circulating sclerostin levels are higher in low muscle mass subjects than healthy controls (Kim et al., 2019). In addition, Huang et al. (2017) found that sclerostin could suppress WNT3a mediated crosstalk between MLO-Y4 osteocytes and muscle cells (C2C12) by regulating the $\mathrm{Wnt} / \beta$-catenin pathway. While a sclerostin inhibitor enhances muscle regeneration, it can also restore muscle function in cancer induced muscle weakness, but it cannot prevent soleus muscle atrophy in rodents after spinal cord injury, possibly because low expression of Lrp5/Lrp6 was observed in the rat soleus (Phillips et al., 2018; Hesse et al., 2019). A detailed mechanism of action regarding muscle function regulation by sclerostin should be explored further.

\section{The effects of sclerostin on bone}

SOST genetic mutation-induced high bone mass was reported in the 1950s, but the pathogenesis of the skeletal disease, named Sclerosteosis, was identified in 2001 (Van Buchem et al., 1955). Moreover, other modifications in or near SOST can cause abnormal BMD, such as Van Buchem disease (Holdsworth et al., 2019). In addition, sclerostin transgenic mice exhibit osteopenia (Winkler et al., 2003). Sclerostin is the second emerging therapeutic target, besides $\mathrm{PTH}$, to promote bone anabolism. Several osteogenic activities, such as mechanical load or intermittent PTH administration, can influence serum sclerostin levels (Keller and Kneissel, 2005; Robling et al., 2008). Besides impairing the canonical Wnt pathway and BMP signaling pathway in bone formation, sclerostin was reported to regulate differentiation and mineralization of osteogenic lineage cells via a matrix extracellular phosphoglycoprotein (MEPE)-acidic serine aspartate-rich MEPE-associated motif 
(ASARM)-dependent mechanism (Atkins et al., 2011). Moreover, labeling osteoblasts with tamoxifen-inducible lineage-tracing strategies, researchers found that sclerostin inhibition could re-activate quiescent bone lining cells, but the underlying mechanism requires further investigation (Kim S.W. et al., 2017). Sclerostin not only affects the activities of osteogenic lineage cells within the bone microenvironment but also promotes bone resorption in a RANKL-dependent pathway in an autocrine manner (Carvalho et al., 2011).

\section{Sclerostin in bone-muscle crosstalk during aging}

Serum sclerostin levels ensue with aging, and osteoclasts are also a source of aging-related sclerostin increase (Mirza et al., 2010). It has been reported that osteoclast cultures from old mice produce more sclerostin than from young mice, suggesting that sclerostin may contribute to decreased bone formation in the elderly (Ota et al., 2013). However, Reppe et al. (2015) demonstrated that osteoporosis patients exhibit lower sclerostin levels than healthy controls. The relationship between sclerostin and bone mass is intriguing, since sclerostin inhibits bone formation and is produced by bone cells. There is a functional negative feedback between sclerostin and formation of mature osteocytes. Furthermore, Chang et al. (2014) found that LRP4 acts as an anchor to trap sclerostin in the local bone environment. Genetic deletion of Lrp4 increases bone mass despite rising serum sclerostin, which indicated that circulating sclerostin level cannot reflect local bone turnover (Chang et al., 2014). Despite the ambiguous relationship between sclerostin level and agedrelated osteoporosis, sclerostin inhibitor was found to increase bone mass and strength in the early stages of treatment in a preclinical study, although the effects were significantly reduced after prolonged treatment. Meanwhile, ablation of Sost increases lean body mass in elderly animals (Kim S.P. et al., 2017). In addition, it has been reported that diabetes is associated with increased risk of sarcopenia. Medeiros et al. (2020) reported on a study involving hemodialysis patients with diabetes and illustrated that sclerostin proportion is higher in patients with type 2 diabetes mellitus compared to healthy controls, possibly indicating that a positive relationship between sclerostin and sarcopenia exists. Thus, sclerostin may play an active role in age-related bonemuscle crosstalk.

\section{OPG/RANKL/RANK}

Receptor Activator of Nuclear Factor кB Ligand, abbreviated as RANKL, is a transmembrane protein located in osteocytes and osteoblasts. When talking about RANKL, it is necessary to introduce the OPG/RANKL/RANK system together. It is well known that bone formation and bone resorption are cyclical, dynamically balanced processes, but the molecular mechanisms that link bone formation and resorption were not substantially improved until 1997 (Simonet et al., 1997; Khosla, 2001). The identification of the OPG/RANKL/RANK system presented a new era in the history of bone biology. RANK is a tumor necrosis factor (TNF) receptor superfamily protein in osteoclasts, it can bind to RANKL to activate major molecules in downstream signaling pathways, such as nuclear factor kappa B (NF- $\mathrm{B}$ ) and colony-stimulating factor 1 receptor, thus driving differentiation of pre-osteoclasts to osteoclasts (Chung et al., 2014). Besides RANK, OPG also binds to RANKL, unlike other receptor proteins, it is a soluble receptor which suppresses osteoclast activation. Dysfunction of the OPG/RANKL/RANK axis can cause osteoporosis (Dufresne et al., 2018). In addition, RANK is reportedly expressed in skeletal muscle, while dysfunction of the OPG/RANKL/RANK axis induces various muscle phenotype, therefore, the OPG/RANKL/RANK axis presents a new avenue for the investigation of bone-muscle interplay (Dufresne et al., 2018).

\section{The effects of OPG/RANKL/RANK on muscle}

RANKL-RANK interaction can activate NF-KB, a proinflammation pathway, which can inhibit myogenic differentiation and enhance the expression of the ubiquitin proteasome system to induce muscle atrophy. Anti-RANKL treatment can shift macrophages into the M2 phenotype and inhibit the activation of NF- $\mathrm{BB}$ in Duchenne muscular dystrophy, thus protecting muscle from chronic inflammation and improving its mechanical properties (Hamoudi et al., 2019). In dystrophic mdx mice, Rank knockout rescues muscle strength, but is less effective than full-length OPG-Fc treatment. This may because while full-length OPG-Fc suppresses the RANK-RANKL combination, it also activates sarco/endoplasmic reticulum $\mathrm{Ca}^{2+}$ ATPase to regulate $\mathrm{Ca}^{2+}$ homeostasis in dystrophic EDL through another pathway (Dufresne et al., 2018). Moreover, Bonnet et al. (2019) observed that RANKL inhibitor Dmab could rescue muscle function in humans. Recently, Hamoudi et al. (2020) reveal that $\mathrm{Opg}$ knockout mice displays selective atrophy of fast-twitch-type IIb myofibers, while $\mathrm{C} 2 \mathrm{C} 12$ myotubes exhibit reduced cross section area when exposed to RANKL, they further confirmed that the OPG/RANKL/RANK axis is involved in muscle function regulation.

\section{The effects of $O P G / R A N K L / R A N K$ on bone}

The study of the OPG/RANKL/RANK system on bone began with the identification of OPG. Simonet et al. (1997) found that OPG is a TNF receptor molecule in the rat intestine, and over-expressing OPG in mice show a predominant decrease in osteoclasts (Khosla, 2001). Hormone deficiency and long-term use of glucocorticoids induced abnormal bone resorption are due to changes in RANKL or OPG expression (Singh et al., 2018). Traditionally, the OPG/RANKL/RANK axis reportedly plays a role in osteoclastogenesis. However, scientists found that OPG/RANKL/RANK has an entirely new role in regulating bone metabolism. Zhang et al. (2016) illustrated that Opg ablated mice show increased adipocytes, while BMSCs from these mice prefer adipogenesis via enhanced expression of adipogenic transcription marker, Ppary. Recently, RANK is found to play a role in osteoclast-to-osteoblast crosstalk via EVs released from osteoclasts to induce reverse RANKL signaling, leading to increased osteogenesis and mineralization (Ikebuchi et al., 2018).

\section{$O P G / R A N K L / R A N K$ in bone-muscle crosstalk during aging}

The imbalance between bone formation and bone resorption leads to age-related osteoporosis (Yang et al., 2019). Much attention has been paid to the role of the OPG/RANKL/RANK axis in this process. Researchers found that RANK, RANKL, 
and RANKL/OPG ratio increase in the bone marrow with age, while the bone protective factor OPG decreases with age (Chung et al., 2014; Zhang et al., 2016). However, Piemontese et al. (2017) found that OPG increase with age in femoral marrow plasma, but it's not as obvious as RANKL, indicating that the RANKL/OPG ratio would increase with age. Furthermore, Singh et al. (2018) demonstrated high serum RANKL/OPG ratio in adult mice, and IL-3 could improve the RANKL/OPG ratio and act as a potent medicine to treat osteoporosis. Therefore, changes in the RANKL/OPG ratio are more appropriate as a determinant of bone mass than individual changes in protein levels, such as RANKL or OPG alone. As for muscle, the relationship between RANKL/OPG/RANK and muscle atrophy has been reported, while the relationship with sarcopenia was rarely reported. Bonnet et al. (2019) observed that RANKL inhibitor Dmab could rescue muscle function in postmenopausal women, as well as in osteo-sarcopenic Pparb ${ }^{-/-}$mice. This suggests that the OPG/RANKL/RANK axis may be an existing and feasible method to treat osteoporosis and sarcopenia together.

\section{SKELETAL MUSCLE-DERIVED EV-microRNAs}

\section{EV-microRNAs of Skeletal Muscle Engaged in Bone-Muscle Crosstalk}

Skeletal muscle, a force generator in motion, also an essential endocrine organ, continually releases myokines and EVs to regulate organic metabolism and homeostasis.

A considerable amount of EVs circulate in the bloodstream, and muscle-derived EVs make up 1-5\% of total EVs in circulation (Guescini et al., 2015). Guescini et al. (2015) illustrated that muscle-specific microRNAs enclosed in EVs are released into the blood during exercise, and $60-65 \%$ of the muscular EVs are CD81 positive. During moderate exercise, contracting muscle can strengthen bone quality and prolong lifespan. However, few studies reported that muscular EVs act on bone. Xu Q. et al. (2018) reported that EVs from C2C12 myoblasts or myotubes promote MC3T3-E1 cell osteogenic differentiation via the $\beta$-catenin signaling pathway, and miR-27a-3p is the major functional factor. Furthermore, miR-27a was found to enhance myoblast differentiation (Huang et al., 2012; Chen et al., 2014) and miR-27a mimic could rescue chronic kidney disease-induced muscle atrophy (Wang et al., 2017). This indicates that miR27a in muscle-derived EVs might function in bone and muscle crosstalk. In addition, besides bone formation, muscular EVs also exert a negative effect on osteoclast formation and its mitochondrial metabolism, the latter is necessary for mature osteoclast resorption (Takafuji et al., 2020).

\section{EV-microRNAs of Skeletal Muscle in Bone-Muscle Crosstalk During Aging}

Intensity and frequency of exercise are reduced in most elderly people, and senescent cell-derived EVs alter their cargo, contributing to age-related decline in somatic function. Alphasarcoglycan, an integral membrane glycoprotein, which specially localized to the sarcolemma acts as a typical marker of muscular EVs (Ervasti et al., 1990; Guescini et al., 2015). Recently, an intriguing study demonstrates that miR-34a is highly expressed in aged mouse skeletal muscle and serum EVs. Furthermore, Fulzele et al. (2019) reported that alpha-sarcoglycan-positive EVs and EVs from oxidative stress treated $\mathrm{C} 2 \mathrm{C} 12$ cells all exhibit that miR-34a increases with age, and these EVs could home to bone marrow, leading to BMSC senescence by repressing SIRT1 expression. Elevated miR-34a in the aging muscle can account for muscle metabolic dysfunction, while decreased SIRT1 activity in aging muscles is associated with impaired muscle performance (Mohamed et al., 2014; Kukreti and Amuthavalli, 2020). Yang et al. (2020) reported that miR-29b-3p is increased in the plasma of aging mice and humans, differentiated atrophic C2C12 myotube could release miR-29b-3p-loaded EVs to induce neuronal dysfunction. miR-29b was shown to contribute to several types of muscle atrophy (Li et al., 2017). Moreover, regarding bone metabolism, miR-29b-3p was found to decrease in osteocytes under mechanical loading. Transfection of miR$29 \mathrm{~b}-3 \mathrm{p}$ in osteocytes reduces IGF-1 production, thus suppressing osteoblast differentiation (Zeng et al., 2019). Future research should investigate whether miR-29b-3p in muscle-derived EVs acts as a critical factor in bone-muscle crosstalk during aging. Research should look into whether muscle-specific microRNAs enclosed in muscular EVs, which are influenced by exercise, such as miR-1, miR-133a, can be used for osteosarcopenia treatment (Guescini et al., 2015).

\section{EV-microRNAs OF BONE CELLS ENGAGED IN BONE-MUSCLE CROSSTALK}

Most scientists focused on the influence of muscle on bone mass, and sometimes on the effects of osteokines on muscle, while no studies directly investigated the influence of EVs secreted by bone on muscle. Herein, we summarize several microRNAs, which appear altered in senescent bone cell-derived EVs, reported to influence bone metabolism and regulate muscle metabolism.

A subset of differentially expressed EV-microRNAs among young and aging BMSCs were found recently (Davis et al., 2017; Umezu et al., 2017; Su et al., 2019). We summarize and select several from 65 age-dependent altered microRNAs, including miR-10a/10b-5p/15b/17$5 \mathrm{p} / 182-5 \mathrm{p} / 183-5 \mathrm{p} / 185 / 186 / 196 \mathrm{a}-5 \mathrm{p} / 20 \mathrm{a} / 221 / 24 / 28-5 \mathrm{p} / 31 \mathrm{a}-$

$5 \mathrm{p} / 328 / 365 / 374 / 411 / 467 \mathrm{e}-5 \mathrm{p} / 487 \mathrm{a} / 99 \mathrm{~b}$ etc. By consulting articles regarding bone metabolism and myogenesis, we screen out four downregulated and seven upregulated microRNAs in aging BMSC-derived exosomes.

The downregulated microRNAs include miR-24, miR-328, miR-365, and miR-374. The commonality between these microRNAs downregulated in aging BMSC-EVs is that they reportedly benefit myogenesis and bone formation, or at least myogenesis. Sun et al. (2008) demonstrated that ectopic expression of miR-24 promotes the expression of myogenic differentiation markers in C2C12 myoblasts. Furthermore, miR365 and miR-374 were reported to accelerate cardiomyocyte 
hypertrophy (Lee et al., 2017; Wu et al., 2017). Besides myogenesis, bone formation is also regulated by these two microRNAs. Concretely, miR-365 overexpression could improve the expression of Runx2 in MC3T3-E1 cells and rescue dexamethasone-induced osteogenesis suppression; it can also suppress bone resorption via decreasing osteoclast number (Franceschetti et al., 2014; Xu et al., 2017). Regarding miR-374, Ge et al. (2018) found that miR-374 expression is significantly increased in fractured mice and verified that miR-374 improves MSC osteogenesis via PTEN using miR-374 transgenic and knockout model. Moreover, miR-328 may play an important role in bone-muscle crosstalk in aging. Liu D. et al. (2018) verified that apoptosis-deficient $M R L / l p r$ and $C a s p 3^{-/-}$mice show impaired osteogenesis capability of BMSCs. Further, they found that circulating apoptotic bodies treatment maintains bone homeostasis of MRL/lpr and Casp3 $3^{-/-}$mice and improves the osteopenia phenotype of ovariectomized mice. Interestingly, Liu D. et al. (2018) found that miR-328 is a functional factor in apoptotic bodies. Lately, miR-328 was reportedly decreased in the plasma of the sarcopenia group in the elderly (Wu et al., 2017).

The upregulated microRNAs include miR-15b, miR-17, miR20a, miR-186, miR-221, miR-31a-5p, and miR-99b. miR-15b was reported to be a negative regulator of myoblast differentiation via SET-domain containing 3 (SETD3) (Zhao et al., 2019). In addition, Fang et al. (2019) found that miR-15b could repress BMSC osteogenesis, while others revealed contrasting result that miR-15b has a positive effect on osteogenic differentiation, therefore, further research is required to determine if miR$15 \mathrm{~b}$ is a potential target. miR-17 and miR-20a belong to the miR-17-92 cluster, which is reported to suppress myogenesis by reducing the expression of enigma homolog 1 (Enh1), then inhibiting myogenic regulatory factor (MRF) and $\mathrm{E}$ protein binding to the myogenesis genes (Qiu et al., 2016; Vimalraj et al., 2014). Moreover, Fang et al. (2016) found that silencing miR17 could improve the micro-architecture of trabecular bones. Meanwhile, miR-17 and miR-20a were reported to enhance BMSC adipogenesis (An et al., 2016; Zhu E. et al., 2018). Nevertheless, Zhou et al. (2014) had shown that miR-1792 cluster heterozygous mice exhibit worse trabecular microarchitecture than wild-type controls. Consequently, scientists should investigate whether miR-17 and miR-20a can act as a cluster enclosed in EVs to influence bone and muscle phenotype together in aging. Combined with the negative effect of miR186 on rat BMSC osteogenesis and the inhibitory action on the final myogenesis differentiation step by influencing myogenin translation, we deduce that miR-186 may be the driving factor in bone-muscle crosstalk during aging (Antoniou et al., 2014; Xiao J. et al., 2020). Furthermore, the increased miR-221 in aged-BMSC may also function in osteosarcopenia, because it was reported that ectopic overexpression of miR-221 in skeletal muscle satellite cells inhibits myotube formation and miR-221 was found to be a negative regulator of bone formation (Zhang et al., 2017; Liu B. et al., 2018; Gan et al., 2020). Xu R. et al. (2018) reported aged BMSCs express high levels of miR-31a$5 p$ leading to bias lineage fate of BMSCs to adipocytes through AT-rich sequence binding protein 2 (SATB2), aged BMSCs also release miR-31a-5p enriched EVs to its microenvironment, inducing increase in osteoclast number and function via the RhoA pathway. Moreover, age-dependent increases in miR-31 can inhibit the dystrophin response to loading, and cause muscles of aged rats more susceptible to injury after disuse (Hughes et al., 2018). Whether these above molecules can participate in age-related bone-muscle crosstalk or not requires further research. Furthermore, we were inspired to investigate whether miR-188, which appears increased in aging BMSCs, can be released through EVs to regulate aging muscle phenotype, since miR-188 was reportedly elevated in a muscular dystrophy dog model (Li et al., 2015; Shibasaki et al., 2019). Zacharewicz et al. $(2014,2020)$ reported that miR-99b displays a negative age-dependent association with protein synthesis in skeletal muscle. Next, they verified that overexpression of miR-99b in human primary myotubes could inhibit protein synthesis via regulatory-associated protein of $\mathrm{mTOR}$ (RPTOR). As for bone, Franceschetti et al. (2014) performed a microRNA array during osteoclastogenesis and found that miR-99b inhibition decreases both osteoclast number and size. Considering the effects of miR$99 \mathrm{~b}$ on muscle and osteoclasts, we hypothesize that miR-99b might become a new target of osteosarcopenia in the elderly.

Besides BMSCs, aged osteoclasts were found to liberate EVs unloading enhanced expression of miR-214-3p comparing with the young. Increasing miR-214-3p not only directly promotes osteoclast formation but it's also enclosed in osteoclast-derived EVs, then leading to impaired bone formation ( $\mathrm{Li} \mathrm{D}$. et al., 2016). However, miR-214 was found to positively regulate $\mathrm{C} 2 \mathrm{C} 12$ myoblasts proliferation and differentiation and reported to be an important participant in muscle metabolism (Feng et al., 2011). Overall, whether miR-214 can serve as a reasonable target to treat age-related osteoporosis and sarcopenia or not needs further exploration. In addition, Davis et al. (2017) collected bone marrow interstitial fluid from mice at two age stages and found that miR-183-5p increases with age in bone-derived EVs and is endocytosed by BMSCs, and finally suppresses osteogenic differentiation and induce BMSCs senescence. However, research is required on its function in muscle metabolism.

\section{CONCLUSION AND FUTURE DIRECTIONS}

There is much literature on bone and muscle crosstalk although, many key issues need to be addressed. Previous studies mainly focused on simple unidirectional communications between bone and muscle, little has reported their age-related reciprocal interactions. We outline age-related changes in the secretome and EV-microRNAs of bone and muscle, and aim to find out their common pathogenic factors and shared therapeutic targets. However, the study of age-related bonemuscle crosstalk is relatively complex, many uncharted fields still need to be explored.

Extracellular vesicles unloading many potential bioactive molecules act as information-transmitters between cells. Besides microRNAs, long non-coding RNA (LncRNA) is also enriched in EVs. No studies have reported its role in bone-muscle crosstalk in 
aging, thus researches on age-related LncRNA changes in BMSCs and muscle, together with their related LncRNA functional studies will provide a niche for further investigation (Nie et al., 2015; Li et al., 2018). Scientists can investigate whether aged bone cells can enclose LncRNA, such as Bmncr, within EVs to regulate musculoskeletal metabolism. Such studies will help to find efficient therapeutic applications in age-related musculoskeletal diseases.

\section{AUTHOR CONTRIBUTIONS}

$\mathrm{CH}$ wrote the manuscript and designed the figures. $\mathrm{WH}, \mathrm{JH}, \mathrm{KC}$, $\mathrm{MH}, \mathrm{MY}$, and XL revised the manuscript. CL provided critical

\section{REFERENCES}

Alibhai, F. J., Lim, F., Yeganeh, A., DiStefano, P. V., Binesh-Marvasti, T., Belfiore, A., et al. (2020). Cellular senescence contributes to age-dependent changes in circulating extracellular vesicle cargo and function. Aging Cell 19:e13103. doi: $10.1111 /$ acel.13103

An, X., Ma, K., Zhang, Z., Zhao, T., Zhang, X., Tang, B., et al. (2016). miR-17, miR-21, and miR-143 enhance adipogenic differentiation from porcine bone marrow-derived mesenchymal stem cells. DNA Cell Biol. 35, 410-416. doi: 10.1089/dna.2015.3182

Antoniou, A., Mastroyiannopoulos, N., Uney, J., and Phylactou, L. (2014). miR-186 inhibits muscle cell differentiation through myogenin regulation. J. Biol. Chem. 289, 3923-3935. doi: 10.1074/jbc.M113.507343

Atkins, G. J., Rowe, P. S., Lim, H. P., Welldon, K. J., Ormsby, R., Wijenayaka, A. R., et al. (2011). Sclerostin is a locally acting regulator of lateosteoblast/preosteocyte differentiation and regulates mineralization through a MEPE-ASARM-dependent mechanism. J. Bone Miner. Res. 26, 1425-1436. doi: $10.1002 / j b m r .345$

Battafarano, G., Rossi, M., Marampon, F., Minisola, S., and Del Fattore, A. (2020). Bone control of muscle function. Int. J. Mol. Sci. 21:1178. doi: 10.3390/ ijms 21041178

Benoit, B., Meugnier, E., Castelli, M., Chanon, S., Vieille-Marchiset, A., Durand, C., et al. (2017). Fibroblast growth factor 19 regulates skeletal muscle mass and ameliorates muscle wasting in mice. Nat. Med. 23, 990-996. doi: 10.1038/nm. 4363

Binkley, N., and Buehring, B. (2009). Beyond FRAX: it's time to consider sarcoosteopenia. J. Clin. Densitomet. 12, 413-416. doi: 10.1016/j.jocd.2009.06.004

Bodine, P. V., and Komm, B. S. (1999). Evidence that conditionally immortalized human osteoblasts express an osteocalcin receptor. Bone 25, 535-543. doi: 10.1016/S8756-3282(99)00213-6

Bonnet, N., Bourgoin, L., Biver, E., Douni, E., and Ferrari, S. (2019). RANKL inhibition improves muscle strength and insulin sensitivity and restores bone mass. J. Clin. Investig. 129, 3214-3223. doi: 10.1172/JCI125915

Boström, P., Wu, J., Jedrychowski, M. P., Korde, A., Ye, L., Lo, J. C., et al. (2012). A PGC1- $\alpha$-dependent myokine that drives brown-fat-like development of white fat and thermogenesis. Nature 481, 463-468. doi: 10.1038/nature10777

Bowser, M., Herberg, S., Arounleut, P., Shi, X., Fulzele, S., Hill, W. D., et al. (2013). Effects of the activin A-myostatin-follistatin system on aging bone and muscle progenitor cells. Exp. Gerontol. 48, 290-297. doi: 10.1016/j.exger.2012.11.004

Carvalho, D. P. D., Wijenayaka, A. R., Kogawa, M., Lim, H. P., Bonewald, L. F., Findlay, D. M., et al. (2011). Sclerostin stimulates osteocyte support of osteoclast activity by a RANKL-dependent pathway. PLoS One 6:e25900. doi: 10.1371/ journal.pone. 025900

Chang, J. S., Kim, T. H., Nguyen, T. T., Park, K.-S., Kim, N., and Kong, I. D. (2017). Circulating irisin levels as a predictive biomarker for sarcopenia: a crosssectional community-based study. Geriatr. Gerontol. Intern. 17, 2266-2273. doi: 10.1111 /ggi.13030

Chang, J. S., and Kong, I. D. (2020). Irisin prevents dexamethasone-induced atrophy in C2C12 myotubes. Pflugers Arch. 472, 495-502. doi: 10.1007/s00424020-02367-4 feedback and helped to shape the manuscript. All authors listed have made a substantial contribution to the work.

\section{FUNDING}

This work was supported by the National Key R\&D Program of China (Grant 2019YFA0111900), the National Natural Science Foundation of China (Grants 81922017, 81873669, and 81802209), the Hunan Province Natural Science Foundation of China (Grant 2018JJ3863), the Innovation Driven Project of Central South University (Grant 20170033010017), and the Talent Plan of Xiangya Hospital in Central South University (Grants 35 and 41).

Chang, M. K., Kramer, I., Huber, T., Kinzel, B., Guth-Gundel, S., Leupin, O., et al. (2014). Disruption of Lrp4 function by genetic deletion or pharmacological blockade increases bone mass and serum sclerostin levels. Proc. Natl. Acad. Sci. U.S.A. 111, E5187-E5195. doi: 10.1073/pnas.1413828111

Charoenphandhu, N., Suntornsaratoon, P., Krishnamra, N., Sa-Nguanmoo, P., Tanajak, P., Wang, X., et al. (2017). Fibroblast growth factor-21 restores insulin sensitivity but induces aberrant bone microstructure in obese insulin-resistant rats. J. Bone Mineral Metab. 35, 142-149. doi: 10.1007/s00774-016-0745-z

Chen, X., Huang, Z., Chen, D., Yang, T., and Liu, G. (2014). Role of microRNA-27a in myoblast differentiation. Cell Biol. Intern. 38, 266-271. doi: 10.1002/cbin. 10192

Chen, Y. S., Guo, Q., Guo, L. J., Liu, T., Wu, X. P., Lin, Z. Y., et al. (2017). GDF8 inhibits bone formation and promotes bone resorption in mice. Clin. Exper. Pharmacol. Physiol. 44, 500-508. doi: 10.1111/1440-1681.12728

Chen, Z., Zhang, Y., Zhao, F., Yin, C., Yang, C., Wang, X., et al. (2020). Recombinant Irisin prevents the reduction of osteoblast differentiation induced by stimulated microgravity through increasing beta-Catenin expression. Int. J. Mol. Sci. 21:1259. doi: 10.3390/ijms21041259

Chung, P. L., Zhou, S., Eslami, B., Shen, L., LeBoff, M. S., and Glowacki, J. (2014). Effect of age on regulation of human osteoclast differentiation. J. Cell. Biochem. 115, 1412-1419. doi: 10.1002/jcb.24792

Cleasby, M. E., Jamieson, P. M., and Atherton, P. J. (2016). Insulin resistance and sarcopenia: mechanistic links between common co-morbidities. J. Endocrinol. 229, R67-R81. doi: 10.1530/JOE-15-0533

Colaianni, G., Cuscito, C., Mongelli, T., Oranger, A., Mori, G., Brunetti, G., et al. (2014). Irisin enhances osteoblast differentiation in vitro. Intern. J. Endocrinol. 2014:902186. doi: 10.1155/2014/902186

Colaianni, G., Cuscito, C., Mongelli, T., Pignataro, P., Buccoliero, C., Liu, P., et al. (2015). The myokine irisin increases cortical bone mass. Proc. Natl. Acad. Sci. U.S.A. 112, 12157-12162. doi: 10.1073/pnas.1516622112

Colaianni, G., Mongelli, T., Cuscito, C., Pignataro, P., Lippo, L., Spiro, G., et al. (2017). Irisin prevents and restores bone loss and muscle atrophy in hind-limb suspended mice. Sci. Rep. 7:2811. doi: 10.1038/s41598-017-02557-8

Dankbar, B., Fennen, M., Brunert, D., Hayer, S., Frank, S., Wehmeyer, C., et al. (2015). Myostatin is a direct regulator of osteoclast differentiation and its inhibition reduces inflammatory joint destruction in mice. Nat. Med. 21, 1085-1090. doi: 10.1038/nm.3917

Das, D. K., Graham, Z. A., and Cardozo, C. P. (2020). Myokines in skeletal muscle physiology and metabolism: recent advances and future perspectives. Acta Physiol. 228:e13367. doi: 10.1111/apha.13367

Davis, C., Dukes, A., Drewry, M., Helwa, I., Johnson, M. H., Isales, C. M., et al. (2017). MicroRNA-183-5p increases with age in bone-derived extracellular vesicles, suppresses bone marrow stromal (Stem) cell proliferation, and induces stem cell senescence. Tissue Eng. Part A 23, 1231-1240. doi: 10.1089/ten.tea. 2016.0525

Delgado-Calle, J., Sato, A. Y., and Bellido, T. (2017). Role and mechanism of action of sclerostin in bone. Bone 96, 29-37. doi: 10.1016/j.bone.2016.10.007

Deng, Z., Luo, P., Lai, W., Song, T., Peng, J., and Wei, H. K. (2017). Myostatin inhibits eEF2K-eEF2 by regulating AMPK to suppress protein synthesis. Biochem. Biophys. Res. Commun. 494, 278-284. doi: 10.1016/j.bbrc.2017.10.040 
Diemar, S. S., Mollehave, L. T., Quardon, N., Lylloff, L., Thuesen, B. H., Linneberg, A., et al. (2020). Effects of age and sex on osteocalcin and bone-specific alkaline phosphatase-reference intervals and confounders for two bone formation markers. Arch. Osteoporos. 15:26. doi: 10.1007/s11657-020-00715-6

Dufresne, S. S., Boulanger-Piette, A., Bosse, S., Argaw, A., Hamoudi, D., Marcadet, L., et al. (2018). Genetic deletion of muscle RANK or selective inhibition of RANKL is not as effective as full-length OPG-fc in mitigating muscular dystrophy. Acta Neuropathol. Commun. 6:31. doi: 10.1186/s40478-018-0533-1

Ervasti, J. M., Ohlendieck, K., Kahl, S. D., Gaver, M. G., and Campbell, K. P. (1990). Deficiency of a glycoprotein component of the dystrophin complex in dystrophic muscle. Nature 345, 315-319. doi: 10.1038/345315a0

Fang, S., Chen, L., Chen, H., Li, Y., Luo, H., Hu, D., et al. (2019). MiR$15 \mathrm{~b}$ ameliorates SONFH by targeting Smad7 and inhibiting osteogenic differentiation of BMSCs. Eur. Rev. Med. Pharmacol. Sci. 23, 9761-9771.

Fang, T., Wu, Q., Zhou, L., Mu, S., and Fu, Q. (2016). miR-106b-5p and miR17-5p suppress osteogenic differentiation by targeting Smad5 and inhibit bone formation. Exper. Cell Res. 347, 74-82. doi: 10.1016/j.yexcr.2016.07.010

Feng, Y., Cao, J. H., Li, X. Y., and Zhao, S. H. (2011). Inhibition of miR-214 expression represses proliferation and differentiation of $\mathrm{C} 2 \mathrm{C} 12$ myoblasts. Cell Biochem. Funct. 29, 378-383. doi: 10.1002/cbf.1760

Fijalkowski, I., Geets, E., Steenackers, E., Van Hoof, V., Ramos, F. J., Mortier, G., et al. (2016). A novel domain-specific mutation in a sclerosteosis patient suggests a role of LRP4 as an anchor for sclerostin in human bone. J. Bone Miner. Res. 31, 874-881. doi: 10.1002/jbmr.2782

Franceschetti, T., Dole, N., Kessler, C., Lee, S., and Delany, A. (2014). Pathway analysis of microRNA expression profile during murine osteoclastogenesis. PLoS One 9:e107262. doi: 10.1371/journal.pone.0107262

Fulzele, S., Mendhe, B., Khayrullin, A., Johnson, M., Kaiser, H., Liu, Y., et al. (2019). Muscle-derived miR-34a increases with age in circulating extracellular vesicles and induces senescence of bone marrow stem cells. Aging 11, 1791-1803. doi: 10.18632/aging.101874

Gan, K., Dong, G., Wang, N., and Zhu, J. (2020). miR-221-3p and miR222-3p downregulation promoted osteogenic differentiation of bone marrow mesenchyme stem cells through IGF-1/ERK pathway under high glucose condition. Diabetes Res. Clin. Pract. 167:108121. doi: 10.1016/j.diabres.2020. 108121

Ge, J., Lin, J., Hong, H., Sun, Y., Li, Y., and Zhang, C. (2018). MiR-374b promotes osteogenic differentiation of MSCs by degrading PTEN and promoting fracture healing. Eur. Rev. Med. Pharmacol. Sci. 22, 3303-3310.

Gong, W., Liu, Y., Wu, Z., Wang, S., Qiu, G., and Lin, S. (2016). Meteorinlike shows unique expression pattern in bone and its overexpression inhibits osteoblast differentiation. PLoS One 11:e0164446. doi: 10.1371/journal.pone. 0164446

Grube, L., Dellen, R., Kruse, F., Schwender, H., Stühler, K., and Poschmann, G. (2018). Mining the secretome of $\mathrm{C} 2 \mathrm{C} 12$ muscle cells: data dependent experimental approach to analyze protein secretion using label-free quantification and peptide based analysis. J. Proteom. Res. 17, 879-890. doi: 10.1021/acs.jproteome.7b00684

Guescini, M., Canonico, B., Lucertini, F., Maggio, S., Annibalini, G., Barbieri, E., et al. (2015). Muscle releases alpha-sarcoglycan positive extracellular vesicles carrying miRNAs in the bloodstream. PLoS One 10:e0125094. doi: 10.1371/ journal.pone.0125094

Hamoudi, D., Bouredji, Z., Marcadet, L., Yagita, H., Landry, L. B., Argaw, A., et al. (2020). Muscle weakness and selective muscle atrophy in osteoprotegerindeficient mice. Hum. Mol. Genet. 29, 483-494. doi: 10.1093/hmg/ddz312

Hamoudi, D., Marcadet, L., Piette Boulanger, A., Yagita, H., Bouredji, Z., Argaw, A., et al. (2019). An anti-RANKL treatment reduces muscle inflammation and dysfunction and strengthens bone in dystrophic mice. Hum. Mol. Genet. 28, 3101-3112. doi: $10.1093 / \mathrm{hmg} / \mathrm{ddz} 124$

Hamrick, M. W., and McGee-Lawrence, M. E. (2018). Blocking bone loss with 1-BAIBA. Trends Endocrinol. Metab. 29, 284-286. doi: 10.1016/j.tem.2018.03. 005

Hamrick, M. W., Shi, X., Zhang, W., Pennington, C., Thakore, H., Haque, M., et al. (2007). Loss of myostatin (GDF8) function increases osteogenic differentiation of bone marrow-derived mesenchymal stem cells but the osteogenic effect is ablated with unloading. Bone 40, 1544-1553. doi: 10.1016/j.bone.2007.02.012

Hanks, L. J., Gutierrez, O. M., Bamman, M. M., Ashraf, A., McCormick, K. L., and Casazza, K. (2015). Circulating levels of fibroblast growth factor-21 increase with age independently of body composition indices among healthy individuals. J. Clin. Transl. Endocrinol. 2, 77-82. doi: 10.1016/j.jcte.2015.02.001

Hao, R. H., Gao, J. L., Li, M., Huang, W., Zhu, D. L., Thynn, H. N., et al. (2018). Association between fibroblast growth factor 21 and bone mineral density in adults. Endocrine 59, 296-303. doi: 10.1007/s12020-017-1507-y

Harslof, T., Sikjaer, T., Mosekilde, L., Langdahl, B. L., and Rejnmark, L. (2016). Correlations between changes in undercarboxylated osteocalcin and muscle function in hypoparathyroidism. Inter. J. Endocrinol. Metab. 14:e38440. doi: $10.5812 / \mathrm{ijem} .38440$

Heredia, J. E., Mukundan, L., Chen, F. M., Mueller, A. A., Deo, R. C., Locksley, R. M., et al. (2013). Type 2 innate signals stimulate fibro/adipogenic progenitors to facilitate muscle regeneration. Cell 153, 376-388. doi: 10.1016/j.cell.2013.02. 053

Hesse, E., Schroder, S., Brandt, D., Pamperin, J., Saito, H., and Taipaleenmaki, H. (2019). Sclerostin inhibition alleviates breast cancer-induced bone metastases and muscle weakness. JCI Insight 5:543. doi: 10.1172/jci.insight. 125543

Holdsworth, G., Roberts, S. J., and Ke, H. Z. (2019). Novel actions of sclerostin on bone. J. Mol. Endocrinol. 62, R167-R185. doi: 10.1530/JME-18-0176

Huang, J., Romero-Suarez, S., Lara, N., Mo, C., Kaja, S., Brotto, L., et al. (2017). Crosstalk between MLO-Y4 osteocytes and C2C12 muscle cells is mediated by the Wnt/beta-catenin pathway. JBMR Plus 1, 86-100. doi: 10.1002/jbm4.10015

Huang, Z., Chen, X., Yu, B., He, J., and Chen, D. (2012). MicroRNA-27a promotes myoblast proliferation by targeting myostatin. Biochem. Biophys. Res. Commun. 423, 265-269. doi: 10.1016/j.bbrc.2012.05.106

Hughes, D. C., Marcotte, G. R., Baehr, L. M., West, D. W. D., Marshall, A. G., Ebert, S. M., et al. (2018). Alterations in the muscle force transfer apparatus in aged rats during unloading and reloading: impact of microRNA-31. J. Physiol. 596, 2883-2900. doi: 10.1113/JP275833

Huh, J. Y., Dincer, F., Mesfum, E., and Mantzoros, C. S. (2014). Irisin stimulates muscle growth-related genes and regulates adipocyte differentiation and metabolism in humans. Int. J. Obes. 38, 1538-1544. doi: 10.1038/ijo.2014.42

Huh, J. Y., Panagiotou, G., Mougios, V., Brinkoetter, M., Vamvini, M. T., Schneider, B. E., et al. (2012). FNDC5 and irisin in humans: I. Predictors of circulating concentrations in serum and plasma and II. mRNA expression and circulating concentrations in response to weight loss and exercise. Metabolism 61, 17251738. doi: 10.1016/j.metabol.2012.09.002

Ibanez, L., Ferrandiz, M. L., Brines, R., Guede, D., Cuadrado, A., and Alcaraz, M. J. (2014). Effects of Nrf2 deficiency on bone microarchitecture in an experimental model of osteoporosis. Oxid. Med. Cell Longev. 2014:726590. doi: 10.1155/2014/ 726590

Ikebuchi, Y., Aoki, S., Honma, M., Hayashi, M., Sugamori, Y., Khan, M., et al. (2018). Coupling of bone resorption and formation by RANKL reverse signalling. Nature 561, 195-200. doi: 10.1038/s41586-018-0482-7

Itoh, N. (2014). FGF21 as a hepatokine, adipokine, and myokine in metabolism and diseases. Front. Endocrinol. 5:107. doi: 10.3389/fendo.2014.00107

Jung, T. W., Hwang, H. J., Hong, H. C., Yoo, H. J., Baik, S. H., and Choi, K. M. (2015). BAIBA attenuates insulin resistance and inflammation induced by palmitate or a high fat diet via an AMPK-PPARdelta-dependent pathway in mice. Diabetologia 58, 2096-2105. doi: 10.1007/s00125-015-3663-z

Kadota, T., Fujita, Y., Yoshioka, Y., Araya, J., Kuwano, K., and Ochiya, T. (2018). Emerging role of extracellular vesicles as a senescence-associated secretory phenotype: Insights into the pathophysiology of lung diseases. Mol. Aspects Med. 60, 92-103. doi: 10.1016/j.mam.2017.11.005

Kammoun, H. L., and Febbraio, M. A. (2014). Come on BAIBA light my fire. Cell Metab. 19, 1-2. doi: 10.1016/j.cmet.2013.12.007

Keller, H., and Kneissel, M. (2005). SOST is a target gene for PTH in bone. Bone 37, 148-158. doi: 10.1016/j.bone.2005.03.018

Khosla, S. (2001). Minireview: the OPG/RANKL/RANK system. Endocrinology 142, 5050-5055. doi: 10.1210/endo.142.12.8536

Kim, H., Wrann, C. D., Jedrychowski, M., Vidoni, S., Kitase, Y., Nagano, K., et al. (2018). Irisin mediates effects on bone and fat via alphaV integrin receptors. Cell 175, 1756-1768.e17. doi: 10.1016/j.cell.2018.10.025

Kim, J. A., Roh, E., Hong, S. H., Lee, Y. B., Kim, N. H., Yoo, H. J., et al. (2019). Association of serum sclerostin levels with low skeletal muscle mass: the Korean Sarcopenic Obesity Study (KSOS). Bone 128:115053. doi: 10.1016/j.bone.2019. 115053

Kim, S. M., Kim, K. M., Kim, B. T., Joo, N. S., Kim, K. N., and Lee, D. J. (2010). Correlation of undercarboxylated osteocalcin (ucOC) concentration and bone 
density with age in healthy Korean women. J. Korea. Med. Sci. 25, 1171-1175. doi: $10.3346 / \mathrm{jkms} .2010 .25 .8 .1171$

Kim, S. P., Frey, J. L., Li, Z., Kushwaha, P., Zoch, M. L., Tomlinson, R. E., et al. (2017). Sclerostin influences body composition by regulating catabolic and anabolic metabolism in adipocytes. Proc. Natl. Acad. Sci. U.S.A. 114, E11238-E11247. doi: 10.1073/pnas.1707876115

Kim, S. W., Lu, Y., Williams, E. A., Lai, F., Lee, J. Y., Enishi, T., et al. (2017), Sclerostin antibody administration converts bone lining cells into active osteoblasts. J. Bone Miner. Res. 32, 892-901. doi: 10.1002/jbmr.3038

Kitase, Y., Vallejo, J. A., Gutheil, W., Vemula, H., Jähn, K., Yi, J., et al. (2018). $\beta$ aminoisobutyric Acid, l-BAIBA, is a muscle-derived osteocyte survival factor. Cell Rep. 22, 1531-1544. doi: 10.1016/j.celrep.2018.01.041

Kukreti, H., and Amuthavalli, K. (2020). MicroRNA-34a causes ceramide accumulation and effects insulin signaling pathway by targeting ceramide kinase (CERK) in aging skeletal muscle. J. Cell. Biochem. 121, 3070-3089. doi: $10.1002 /$ jcb. 29312

Lee, J., Park, J., Kim, Y. H., Lee, N. H., and Song, K. M. (2019). Irisin promotes C2C12 myoblast proliferation via ERK-dependent CCL7 upregulation. PLoS One 14:e0222559. doi: 10.1371/journal.pone.0222559

Lee, J., Song, D., Park, J., Kim, J., Cho, C., and Kim, D. (2017). miR-374 promotes myocardial hypertrophy by negatively regulating vascular endothelial growth factor receptor-1 signaling. BMB Rep. 50, 208-213. doi: 10.5483/BMBRep.2017. 50.4.165

Lee, N. K., Sowa, H., Hinoi, E., Ferron, M., Ahn, J. D., Confavreux, C., et al. (2007). Endocrine regulation of energy metabolism by the skeleton. Cell 130, 456-469. doi: $10.1016 /$ j.cell.2007.05.047

Lee, S. Y., Fam, K. D., Chia, K. L., Yap, M. M. C., Goh, J., Yeo, K. P., et al. (2020). Age-related bone loss is associated with FGF21 but not IGFBP1 in healthy adults. Exper. Physiol. 105, 622-631. doi: 10.1113/EP088351

Levinger, I., Scott, D., Nicholson, G. C., Stuart, A. L., Duque, G., McCorquodale, T., et al. (2014). Undercarboxylated osteocalcin, muscle strength and indices of bone health in older women. Bone 64, 8-12. doi: 10.1016/j.bone.2014.03.008

Li, C. J., Cheng, P., Liang, M. K., Chen, Y. S., Lu, Q., Wang, J. Y., et al. (2015). MicroRNA-188 regulates age-related switch between osteoblast and adipocyte differentiation. J. Clin. Invest. 125, 1509-1522. doi: 10.1172/JCI77716

Li, C. J., Xiao, Y., Yang, M., Su, T., Sun, X., Guo, Q., et al. (2018). Long noncoding RNA Bmncr regulates mesenchymal stem cell fate during skeletal aging. J. Clin. Invest. 128, 5251-5266. doi: 10.1172/JCI99044

Li, D., Liu, J., Guo, B., Liang, C., Dang, L., Lu, C., et al. (2016). Osteoclast-derived exosomal miR-214-3p inhibits osteoblastic bone formation. Nat. Commun. 7:10872. doi: $10.1038 /$ ncomms 10872

Li, J., Zhang, H., Yang, C., Li, Y., and Dai, Z. (2016). An overview of osteocalcin progress. J. Bone Miner. Metab. 34, 367-379. doi: 10.1007/s00774-015-0734-7

Li, G., Zhang, L., Wang, D., AIQudsy, L., Jiang, J. X., Xu, H., et al. (2019). Musclebone crosstalk and potential therapies for sarco-osteoporosis. J.Cell. Biochem. 120, 14262-14273. doi: $10.1002 / j c b .28946$

Li, J., Chan, M., Yu, Y., Bei, Y., Chen, P., Zhou, Q., et al. (2017). miR-29b contributes to multiple types of muscle atrophy. Nat. Commun. 8:15201. doi: $10.1038 /$ ncomms 15201

Li, Z.-Y., Zheng, S.-L., Wang, P., Xu, T.-Y., Guan, Y.-F., Zhang, Y.-J., et al. (2014). Subfatin is a novel adipokine and unlike meteorin in adipose and brain expression. CNS Neurosci. Therapeut. 20, 344-354. doi: 10.1111/cns. 12219

Lin, X., Hanson, E., Betik, A. C., Brennan-Speranza, T. C., Hayes, A., and Levinger, I. (2016). Hindlimb immobilization, but not castration, induces reduction of undercarboxylated osteocalcin associated with muscle atrophy in rats. J. Bone Miner. Res. 31, 1967-1978. doi: 10.1002/jbmr.2884

Ling, Y., Gao, X., Lin, H., Ma, H., Pan, B., and Gao, J. (2016). A common polymorphism rs1800247 in osteocalcin gene was associated with serum osteocalcin levels, bone mineral density, and fracture: the Shanghai Changfeng Study. Osteoporos. Intern. 27, 769-779. doi: 10.1007/s00198-015-3244-5

Liu, B., Shi, Y., He, H., Cai, M., Xiao, W., Yang, X., et al. (2018). miR-221 modulates skeletal muscle satellite cells proliferation and differentiation. Vitro Cell. Dev. Biol. Anim. 54, 147-155. doi: 10.1007/s11626-017-0210-x

Liu, D., Kou, X., Chen, C., Liu, S., Liu, Y., Yu, W., et al. (2018). Circulating apoptotic bodies maintain mesenchymal stem cell homeostasis and ameliorate osteopenia via transferring multiple cellular factors. Cell Res. 28, 918-933. doi: 10.1038/s41422-018-0070-2
Liu, S., Gao, F., Wen, L., Ouyang, M., Wang, Y., Wang, Q., et al. (2017). Osteocalcin induces proliferation via positive activation of the PI3K/Akt, P38 MAPK pathways and promotes differentiation through activation of the GPRC6A-ERK1/2 pathway in C2C12 myoblast cells. Cell. Physiol. Biochem. 43, 1100-1112. doi: $10.1159 / 000481752$

Liu, X., Wang, Y., Hou, L., Xiong, Y., and Zhao, S. (2017a). Fibroblast growth factor 21 (FGF21) promotes formation of aerobic myofibers via the FGF21-SIRT1AMPK-PGC1alpha pathway. J. Cell. Physiol. 232, 1893-1906. doi: 10.1002/jcp. 25735

Liu, X., Wang, Y., Zhao, S., and Li, X. (2017b). Fibroblast growth factor 21 promotes $\mathrm{C} 2 \mathrm{C} 12$ cells myogenic differentiation by enhancing cell cycle exit. Biomed. Res. Int. 2017:1648715. doi: 10.1155/2017/1648715

Ma, Y., Qiao, X., Zeng, R., Cheng, R., Zhang, J., Luo, Y., et al. (2018). Irisin promotes proliferation but inhibits differentiation in osteoclast precursor cells. FASEB J. 32, 5813-5823. doi: 10.1096/fj.201700983RR

Mahmoodnia, L., Sadoughi, M., Ahmadi, A., and Kafeshani, M. (2016). Relationship between serum irisin, glycemic indices, and renal function in type 2 diabetic patients. J. Ren. Injury Prevent. 6, 88-92. doi: 10.15171/jrip.2017.17

McPherron, A. C., Lawler, A. M., and Lee, S. J. (1997). Regulation of skeletal muscle mass in mice by a new TGF-beta superfamily member. Nature $387,83-90$. doi: $10.1038 / 387083 \mathrm{a} 0$

Medeiros, M. C., Rocha, N., Bandeira, E., Dantas, I., Chaves, C., Oliveira, M., et al. (2020). Serum sclerostin, body composition, and sarcopenia in hemodialysis patients with diabetes. Intern. J. Nephrol. 2020:4596920. doi: 10.1155/2020/ 4596920

Mera, P., Laue, K., Ferron, M., Confavreux, C., Wei, J., Galán-Díez, M., et al. (2016a). Osteocalcin signaling in myofibers is necessary and sufficient for optimum adaptation to exercise. Cell Metab. 23, 1078-1092. doi: 10.1016/j. cmet.2016.05.004

Mera, P., Laue, K., Wei, J., Berger, J. M., and Karsenty, G. (2016b). Osteocalcin is necessary and sufficient to maintain muscle mass in older mice. Mol. Metab. 5, 1042-1047. doi: 10.1016/j.molmet.2016.07.002

Mirza, F. S., Padhi, I. D., Raisz, L. G., and Lorenzo, J. A. (2010). Serum sclerostin levels negatively correlate with parathyroid hormone levels and free estrogen index in postmenopausal women. J. Clin. Endocrinol. Metab. 95, 1991-1997. doi: $10.1210 /$ jc. $2009-2283$

Mizokami, A., Kawakubo-Yasukochi, T., and Hirata, M. (2017). Osteocalcin and its endocrine functions. Biochem. Pharmacol. 132, 1-8. doi: 10.1016/j.bcp.2017.02. 001

Mohamed, J. S., Wilson, J. C., Myers, M. J., Sisson, K. J., and Alway, S. E. (2014). Dysregulation of SIRT-1 in aging mice increases skeletal muscle fatigue by a PARP-1-dependent mechanism. Aging 6, 820-834. doi: 10.18632/aging.100696

Morissette, M. R., Stricker, J. C., Rosenberg, M. A., Buranasombati, C., Levitan, E. B., Mittleman, M. A., et al. (2009). Effects of myostatin deletion in aging mice. Aging Cell 8, 573-583. doi: 10.1111/j.1474-9726.2009.00508.x

Neve, A., Corrado, A., and Cantatore, F. P. (2013). Osteocalcin: skeletal and extra-skeletal effects. J. Cell. Physiol. 228, 1149-1153. doi: 10.1002/jcp.24278

Nie, M., Deng, Z. L., Liu, J., and Wang, D. Z. (2015). Noncoding RNAs, emerging regulators of skeletal muscle development and diseases. Biomed. Res. Intern. 2015:676575. doi: 10.1155/2015/676575

Oost, L. J., Kustermann, M., Armani, A., Blaauw, B., and Romanello, V. (2019). Fibroblast growth factor 21 controls mitophagy and muscle mass. J. Cachexia Sarcopenia Muscle 10, 630-642. doi: 10.1002/jcsm.12409

Ota, K., Quint, P., Ruan, M., Pederson, L., Westendorf, J. J., Khosla, S., et al. (2013). Sclerostin is expressed in osteoclasts from aged mice and reduces osteoclastmediated stimulation of mineralization. J. Cell. Biochem. 114, 1901-1907. doi: $10.1002 /$ jcb. 24537

Perie, L., Parente, A., Brun, C., Magnol, L., Pelissier, P., and Blanquet, V. (2016). Enhancement of $\mathrm{C} 2 \mathrm{C} 12$ myoblast proliferation and differentiation by GASP-2, a myostatin inhibitor. Biochem. Biophys. Rep. 6, 39-46. doi: 10.1016/j.bbrep. 2016.03.001

Phillips, E. G., Beggs, L. A., Ye, F., Conover, C. F., Beck, D. T., Otzel, D. M., et al. (2018). Effects of pharmacologic sclerostin inhibition or testosterone administration on soleus muscle atrophy in rodents after spinal cord injury. PLoS One 13:e0194440. doi: 10.1371/journal.pone.0194440

Piemontese, M., Almeida, M., Robling, A. G., Kim, H. N., Xiong, J., Thostenson, J. D., et al. (2017). Old age causes de novo intracortical bone remodeling and porosity in mice. JCI Insight 2:e93771. doi: 10.1172/jci.insight.93771 
Poggioli, T., Vujic, A., Yang, P., Macias-Trevino, C., Uygur, A., Loffredo, F. S., et al. (2016). Circulating growth differentiation factor 11/8 levels decline with age. Circ. Res. 118, 29-37. doi: 10.1161/CIRCRESAHA.115.307521

Puig, J., Blasco, G., Daunis-i-Estadella, J., Moreno, M., Molina, X., AlberichBayarri, A., et al. (2016). Lower serum osteocalcin concentrations are associated with brain microstructural changes and worse cognitive performance. Clin. Endocrinol. 84, 756-763. doi: 10.1111/cen.12954

Qiao, X., Nie, Y., Ma, Y., Chen, Y., Cheng, R., Yin, W., et al. (2016). Irisin promotes osteoblast proliferation and differentiation via activating the MAP kinase signaling pathways. Sci. Rep. 6:18732. doi: 10.1038/srep21053

Qin, Y., Peng, Y., Zhao, W., Pan, J., Ksiezak-Reding, H., Cardozo, C., et al. (2017). Myostatin inhibits osteoblastic differentiation by suppressing osteocyte-derived exosomal microRNA-218: a novel mechanism in muscle-bone communication. J. Biol. Chem. 292, 11021-11033. doi: 10.1074/jbc.M116.770941

Qiu, H., Liu, N., Luo, L., Zhong, J., Tang, Z., Kang, K., et al. (2016). MicroRNA17-92 regulates myoblast proliferation and differentiation by targeting the ENH1/Id1 signaling axis. Cell Death Differ. 23, 1658-1669. doi: 10.1038/cdd. 2016.56

Ran, N., Gao, X., Dong, X., Li, J., Lin, C., Geng, M., et al. (2020). Effects of exosomemediated delivery of myostatin propeptide on functional recovery of mdx mice. Biomaterials 236:119826. doi: 10.1016/j.biomaterials.2020.119826

Rana, K. S., Arif, M., Hill, E. J., Aldred, S., Nagel, D. A., Nevill, A., et al. (2014). Plasma irisin levels predict telomere length in healthy adults. Age 36, 995-1001. doi: 10.1007/s11357-014-9620-9

Rao, R. R., Long, J. Z., White, J. P., Svensson, K. J., Lou, J., Lokurkar, I., et al. (2014). Meteorin-like is a hormone that regulates immune-adipose interactions to increase beige fat thermogenesis. Cell 157, 1279-1291. doi: 10.1016/j.cell. 2014.03.065

Rebbapragada, A., Benchabane, H., Wrana, J. L., Celeste, A. J., and Attisano, L. (2003). Myostatin signals through a transforming growth factor beta-like signaling pathway to block adipogenesis. Mol. Cell. Biol. 23, 7230-7242. doi: 10.1128/MCB.23.20.7230-7242.2003

Reginster, J. Y., Beaudart, C., Buckinx, F., and Bruyère, O. (2016). Osteoporosis and sarcopenia: two diseases or one? Curr. Opin. Clin. Nutr. Metab. Care 19, 31-36. doi: 10.1097/MCO.0000000000000230

Reppe, S., Noer, A., Grimholt, R. M., Halldórsson, B. V., Medina-Gomez, C., Gautvik, V. T., et al. (2015). Methylation of bone SOST, its mRNA, and serum sclerostin levels correlate strongly with fracture risk in postmenopausal women. J. Bone Miner. Res. 30, 249-256. doi: 10.1002/jbmr.2342

Reza, M. M., Sim, C. M., Subramaniyam, N., Ge, X., Sharma, M., Kambadur, R., et al. (2017a). Irisin treatment improves healing of dystrophic skeletal muscle. Oncotarget 8, 98553-98566. doi: 10.18632/oncotarget.21636

Reza, M. M., Subramaniyam, N., Sim, C. M., Ge, X., Sathiakumar, D., McFarlane, C., et al. (2017b). Irisin is a pro-myogenic factor that induces skeletal muscle hypertrophy and rescues denervation-induced atrophy. Nat. Commun. 8:1104. doi: 10.1038/s41467-017-01131-0

Roberts, L. D., Bostrom, P., O’Sullivan, J. F., Schinzel, R. T., Lewis, G. D., Dejam, A., et al. (2014). beta-Aminoisobutyric acid induces browning of white fat and hepatic beta-oxidation and is inversely correlated with cardiometabolic risk factors. Cell Metab. 19, 96-108. doi: 10.1016/j.cmet.2013.12.003

Robling, A. G., Niziolek, P. J., Baldridge, L. A., Condon, K. W., Allen, M. R., Alam, I., et al. (2008). Mechanical stimulation of bone in vivo reduces osteocyte expression of Sost/sclerostin. J. Biol. Chem. 283, 5866-5875. doi: 10.1074/jbc. M705092200

Rodriguez, J., Vernus, B., Chelh, I., Cassar-Malek, I., Gabillard, J. C., Hadj Sassi, A., et al. (2014). Myostatin and the skeletal muscle atrophy and hypertrophy signaling pathways. Cell Mol. Life Sci. 71, 4361-4371. doi: 10.1007/s00018-0141689-x

Ruan, Q., Huang, Y., Yang, L., Ruan, J., Gu, W., Zhang, X., et al. (2019). The effects of both age and sex on irisin levels in paired plasma and cerebrospinal fluid in healthy humans. Peptides 113, 41-51. doi: 10.1016/j.peptides.2019.01.004

Severinsen, M. C. K., and Pedersen, B. K. (2020). Muscle-organ crosstalk: the emerging roles of myokines. Endocr. Rev. 41, 594-609. doi: 10.1210/endrev/ bnaa016

Shan, T., Liang, X., Bi, P., and Kuang, S. (2013). Myostatin knockout drives browning of white adipose tissue through activating the AMPK-PGC1alphaFndc5 pathway in muscle. FASEB J. 27, 1981-1989. doi: 10.1096/fj.12-225755

Shibaguchi, T., Maeoka, T., Yoshihara, T., Naito, H., Goto, K., Yoshioka, T., et al. (2018). Age-related changes in myostatin expression in rat skeletal muscles. J. Phys. Fitness Sports Med. 7, 221-227. doi: 10.7600/jpfsm. 7.221

Shibasaki, H., Imamura, M., Arima, S., Tanihata, J., Kuraoka, M., Matsuzaka, Y., et al. (2019). Characterization of a novel microRNA, miR-188, elevated in serum of muscular dystrophy dog model. PLoS One 14:e0211597. doi: 10.1371/journal. pone. 0211597

Simonet, W. S., Lacey, D. L., Dunstan, C. R., Kelley, M., Chang, M. S., Lüthy, R., et al. (1997). Osteoprotegerin: a novel secreted protein involved in the regulation of bone density. Cell 89, 309-319. doi: 10.1016/S0092-8674(00) 80209-3

Singh, K., Piprode, V., Mhaske, S. T., Barhanpurkar-Naik, A., and Wani, M. R. (2018). IL-3 differentially regulates membrane and soluble rankl in osteoblasts through metalloproteases and the JAK2/STAT5 pathway and improves the RANKL/OPG ratio in adult mice. J. Immunol. 200, 595-606. doi: 10.4049/ jimmunol.1601528

Siriett, V., Platt, L., Salerno, M. S., Ling, N., Kambadur, R., and Sharma, M. (2006). Prolonged absence of myostatin reduces sarcopenia. J. Cell. Physiol. 209, 866-873. doi: $10.1002 /$ jcp. 20778

Steensberg, A., van Hall, G., Osada, T., Sacchetti, M., Saltin, B., and Klarlund Pedersen, B. (2000). Production of interleukin-6 in contracting human skeletal muscles can account for the exercise-induced increase in plasma interleukin-6. J. Physiol. 529(Pt 1), 237-242. doi: 10.1111/j.1469-7793.2000.00237.x

Storlino, G., Colaianni, G., Sanesi, L., Lippo, L., Brunetti, G., Errede, M., et al. (2020). Irisin prevents disuse-induced osteocyte apoptosis. J. Bone Miner. Res. 35, 766-775. doi: 10.1002/jbmr.3944

Su, T., Xiao, Y., Xiao, Y., Guo, Q., Li, C., Huang, Y., et al. (2019). Bone marrow mesenchymal stem cells-derived exosomal MiR-29b-3p regulates aging-associated insulin resistance. ACS Nano 13, 2450-2462. doi: 10.1021/ acsnano.8b09375

Sun, L., Sun, S., Zhao, X., Zhang, J., Guo, J., Tang, L., et al. (2019). Inhibition of myostatin signal pathway may be involved in low-intensity pulsed ultrasound promoting bone healing. J. Med. Ultrason. 46, 377-388. doi: 10.1007/s10396019-00962-2

Sun, Q., Zhang, Y., Yang, G., Chen, X., Zhang, Y., Cao, G., et al. (2008). Transforming growth factor-beta-regulated miR-24 promotes skeletal muscle differentiation. Nucleic Acids Res. 36, 2690-2699. doi: 10.1093/nar/ gkn032

Szulc, P., Schoppet, M., Goettsch, C., Rauner, M., Dschietzig, T., Chapurlat, R., et al. (2012). Endocrine and clinical correlates of myostatin serum concentration in men-the STRAMBO study. J. Clin. Endocrinol. Metab. 97, 3700-3708. doi: 10.1210/jc.2012-1273

Takafuji, Y., Tatsumi, K., Ishida, M., Kawao, N., Okada, K., and Kaji, H. (2020). Extracellular vesicles secreted from mouse muscle cells suppress osteoclast formation: roles of mitochondrial energy metabolism. Bone 134:115298. doi: 10.1016/j.bone. 2020.115298

Tezze, C., Romanello, V., Desbats, M. A., Fadini, G. P., Albiero, M., Favaro, G., et al. (2017). Age-associated loss of OPA1 in muscle impacts muscle mass, metabolic homeostasis, systemic inflammation, and epithelial senescence. Cell Metab. 25, 1374-1389.e6. doi: 10.1016/j.cmet.2017.04.021

Umezu, T., Imanishi, S., Azuma, K., Kobayashi, C., Yoshizawa, S., Ohyashiki, K., et al. (2017). Replenishing exosomes from older bone marrow stromal cells with miR-340 inhibits myeloma-related angiogenesis. Blood Adv. 1, 812-823. doi: 10.1182/bloodadvances.2016003251

Ushach, I., Arrevillaga-Boni, G., Heller, G. N., Pone, E., Hernandez-Ruiz, M., Catalan-Dibene, J., et al. (2018). Meteorin-like/Meteorin-beta is a novel immunoregulatory cytokine associated with inflammation. J. Immunol. 201, 3669-3676. doi: 10.4049/jimmunol.1800435

Van Buchem, F. S., Hadders, H. N., and Ubbens, R. (1955). An uncommon familial systemic disease of the skeleton: hyperostosis corticalis generalisata familiaris. Acta Radiol. 44, 109-120. doi: 10.3109/00016925509170789

Vaynman, S., Ying, Z., and Gomez-Pinilla, F. (2004). Hippocampal BDNF mediates the efficacy of exercise on synaptic plasticity and cognition. Eur. J. Neurosci. 20, 2580-2590. doi: 10.1111/j.1460-9568.2004.03720.x

Vimalraj, S., Partridge, N., and Selvamurugan, N. (2014). A positive role of microRNA-15b on regulation of osteoblast differentiation. J. Cell. Physiol. 229, 1236-1244. doi: 10.1002/jcp.24557

von Maltzahn, J., Chang, N. C., Bentzinger, C. F., and Rudnicki, M. A. (2012). Wnt signaling in myogenesis. Trends Cell Biol. 22, 602-609. doi: 10.1016/j.tcb.2012. 07.008 
Wang, B., Zhang, C., Zhang, A., Cai, H., Price, S. R., and Wang, X. H. (2017). MicroRNA-23a and MicroRNA-27a mimic exercise by ameliorating CKDInduced muscle atrophy. J. Am. Soc. Nephrol. 28, 2631-2640. doi: 10.1681/ASN. 2016111213

Wang, D. T., Yang, Y. J., Huang, R. H., Zhang, Z. H., and Lin, X. (2015). Myostatin activates the ubiquitin-proteasome and autophagy-lysosome systems contributing to muscle wasting in chronic kidney disease. Oxid. Med. Cell Longev. 2015:684965. doi: 10.1155/2015/684965

Wang, X., Wei, W., Krzeszinski, J. Y., Wang, Y., and Wan, Y. (2015). A Liver-bone endocrine relay by IGFBP1 promotes osteoclastogenesis and mediates FGF21induced bone resorption. Cell Metab. 22, 811-824. doi: 10.1016/j.cmet.2015.09. 010

Wang, Z., Bian, L., Mo, C., Shen, H., Zhao, L. J., Su, K. J., et al. (2020). Quantification of aminobutyric acids and their clinical applications as biomarkers for osteoporosis. Commun. Biol. 3:39. doi: 10.1038/s42003-0200766-y

Wei, W., Dutchak, P. A., Wang, X., Ding, X., Wang, X., Bookout, A. L., et al. (2012). Fibroblast growth factor 21 promotes bone loss by potentiating the effects of peroxisome proliferator-activated receptor gamma. Proc. Natl. Acad. Sci. U.S.A. 109, 3143-3148. doi: 10.1073/pnas.1200797109

Weilner, S., Schraml, E., Wieser, M., Messner, P., Schneider, K., Wassermann, K., et al. (2016). Secreted microvesicular miR-31 inhibits osteogenic differentiation of mesenchymal stem cells. Aging Cell 15, 744-754. doi: 10.1111/acel.12484

White, T. A., and LeBrasseur, N. K. (2014). Myostatin and sarcopenia: opportunities and challenges - a mini-review. Gerontology 60, 289-293. doi: $10.1159 / 000356740$

Winkler, D. G., Sutherland, M. K., Geoghegan, J. C., Yu, C., Hayes, T., Skonier, J. E., et al. (2003). Osteocyte control of bone formation via sclerostin, a novel BMP antagonist. EMBO J. 22, 6267-6276. doi: 10.1093/emboj/cdg599

Wu, H., Wang, Y., Wang, X., Li, R., and Yin, D. (2017). MicroRNA-365 accelerates cardiac hypertrophy by inhibiting autophagy via the modulation of Skp2 expression. Biochem. Biophys. Res. Commun. 484, 304-310. doi: 10.1016/j.bbrc. 2017.01.108

Wu, L. F., Zhu, D. C., Wang, B. H., Lu, Y. H., He, P., Zhang, Y. H., et al. (2018). Relative abundance of mature myostatin rather than total myostatin is negatively associated with bone mineral density in Chinese. J. Cell. Mol. Med. 22, 1329-1336. doi: $10.1111 /$ jcmm.13438

Xiao, J., Qin, S., Li, W., Yao, L., Huang, P., Liao, J., et al. (2020). Osteogenic differentiation of rat bone mesenchymal stem cells modulated by MiR-186 via SIRT6. Life Sci. 253:117660. doi: 10.1016/j.lfs.2020.117660

Xiao, Y. Z., Yang, M., Xiao, Y., Guo, Q., Huang, Y., Li, C. J., et al. (2020). Reducing hypothalamic stem cell senescence protects against aging-associated physiological decline. Cell Metab. 31, 534-548.e5. doi: 10.1016/j.cmet.2020.01. 002

Xin, C., Liu, J., Zhang, J., Zhu, D., Wang, H., Xiong, L., et al. (2016). Irisin improves fatty acid oxidation and glucose utilization in type 2 diabetes by regulating the AMPK signaling pathway. Intern. J. Obes. 40, 443-451. doi: 10.1038/ijo.2015. 199

Xu, D., Gao, Y., Hu, N., Wu, L., and Chen, Q. (2017). miR-365 ameliorates dexamethasone-induced suppression of osteogenesis in MC3T3-E1 cells by targeting HDAC4. Intern. J. Mol. Sci. 18:977. doi: 10.3390/ijms18050977

Xu, L., Shen, L., Yu, X., Li, P., Wang, Q., and Li, C. (2020). Effects of irisin on osteoblast apoptosis and osteoporosis in postmenopausal osteoporosis rats through upregulating Nrf2 and inhibiting NLRP3 inflammasome. Exp. Ther. Med. 19, 1084-1090. doi: 10.3892/etm.2019.8313

Xu, Q., Cui, Y., Luan, J., Zhou, X., Li, H., and Han, J. (2018). Exosomes from C2C12 myoblasts enhance osteogenic differentiation of MC3T3-E1 pre-osteoblasts by delivering miR-27a-3p. Biochem. Biophys. Res. Commun. 498, 32-37. doi: 10. 1016/j.bbrc.2018.02.144

Xu, R., Shen, X., Si, Y., Fu, Y., Zhu, W., Xiao, T., et al. (2018). MicroRNA31a-5p from aging BMSCs links bone formation and resorption in the aged bone marrow microenvironment. Aging Cell 17:e12794. doi: 10.1111/acel. 12794

Yan, J., Liu, H. J., Guo, W. C., and Yang, J. (2018). Low serum concentrations of Irisin are associated with increased risk of hip fracture in Chinese older women. Joint Bone Spine 85, 353-358. doi: 10.1016/j.jbspin.2017.03.011

Yang, C., Yang, W., Wong, Y., Wang, K., Teng, Y., Chang, M., et al. (2020). Muscle atrophy-related myotube-derived exosomal microRNA in neuronal dysfunction: Targeting both coding and long noncoding RNAs. Aging Cell 19:e13107. doi: 10.1111/acel.13107

Yang, M., Guo, Q., Peng, H., Xiao, Y. Z., Xiao, Y., Huang, Y., et al. (2019). Kruppellike factor 3 inhibition by mutated lncRNA Reglcp results in human high bone mass syndrome. J. Exp. Med. 216, 1944-1964. doi: 10.1084/jem.20181554

Yang, M., Li, C. J., Sun, X., Guo, Q., Xiao, Y., Su, T., et al. (2017). MiR497195 cluster regulates angiogenesis during coupling with osteogenesis by maintaining endothelial Notch and HIF-1 $\alpha$ activity. Nat. Commun. 8:16003. doi: $10.1038 /$ ncomms 16003

Ye, W., Wang, J., Lin, D., and Ding, Z. (2020). The immunomodulatory role of irisin on osteogenesis via AMPK-mediated macrophage polarization. Int. J. Biol. Macromol. 146, 25-35. doi: 10.1016/j.ijbiomac.2019.12.028

Zacharewicz, E., Della Gatta, P., Reynolds, J., Garnham, A., Crowley, T., Russell, A., et al. (2014). Identification of microRNAs linked to regulators of muscle protein synthesis and regeneration in young and old skeletal muscle. PLoS One 9:e114009. doi: 10.1371/journal.pone.0114009

Zacharewicz, E., Kalanon, M., Murphy, R., Russell, A., and Lamon, S. (2020). MicroRNA-99b-5p downregulates protein synthesis in human primary myotubes. Am. J. Physiol. Cell Physiol. 319, C432-C440. doi: 10.1152/ajpcell. 00172.2020

Zeng, Q., Wang, Y., Gao, J., Yan, Z., Li, Z., Zou, X., et al. (2019). miR29b-3p regulated osteoblast differentiation via regulating IGF-1 secretion of mechanically stimulated osteocytes. Cell. Mol. Biolo. Lett. 24:11. doi: 10.1186/ s11658-019-0136-2

Zhang, L., Liu, M., Zhou, X., Liu, Y., Jing, B., Wang, X., et al. (2016). Role of osteoprotegerin (OPG) in bone marrow adipogenesis. Cell. Physiol. Biochem. 40, 681-692. doi: 10.1159/000452580

Zhang, N., Chim, Y. N., Wang, J., Wong, R. M. Y., Chow, S. K. H., and Cheung, W. H. (2020). Impaired fracture healing in sarco-osteoporotic mice can be rescued by vibration treatment through myostatin suppression. J. Orthop. Res. 38, 277-287. doi: 10.1002/jor.24477

Zhang, Y., Gao, Y., Cai, L., Li, F., Lou, Y., Xu, N., et al. (2017). MicroRNA-221 is involved in the regulation of osteoporosis through regulates RUNX2 protein expression and osteoblast differentiation. Am. J. Transl. Res. 9, 126-135.

Zhao, M., Xie, J., Shu, W., Wang, H., Bi, J., Jiang, W., et al. (2019). MiR-15b and miR-322 inhibit SETD3 expression to repress muscle cell differentiation. Cell Death Dis. 10:183. doi: 10.1038/s41419-019-1432-5

Zhong, N., Xu, B., Cui, R., Xu, M., Su, J., Zhang, Z., et al. (2016). Positive correlation between serum osteocalcin and testosterone in male hyperthyroidism patients with high bone turnover. Exper. Clin. Endocrinol. Diabetes 124, 452-456. doi: $10.1055 / \mathrm{s}-0042-107944$

Zhou, L., Song, H. Y., Gao, L. L., Yang, L. Y., Mu, S., and Fu, Q. (2019). MicroRNA100-5p inhibits osteoclastogenesis and bone resorption by regulating fibroblast growth factor 21. Intern. J. Mol. Med. 43, 727-738. doi: 10.3892/ijmm.2018. 4017

Zhou, M., Ma, J., Chen, S., Chen, X., and Yu, X. (2014). MicroRNA-17-92 cluster regulates osteoblast proliferation and differentiation. Endocrine 45, 302-310. doi: 10.1007/s12020-013-9986-y

Zhu, E., Zhang, J., Zhou, J., Yuan, H., Zhao, W., and Wang, B. (2018). miR$20 \mathrm{a}-5 \mathrm{p}$ promotes adipogenic differentiation of murine bone marrow stromal cells via targeting Kruppel-like factor 3. J. Mol. Endocrinol. 60, 225-237. doi: 10.1530/JME-17-0183

Zhu, X. W., Ding, K., Dai, X. Y., and Ling, W. Q. (2018). beta-aminoisobutyric acid accelerates the proliferation and differentiation of MC3T3-E1 cells via moderate activation of ROS signaling. J. Chin. Med. Assoc. 81, 611-618. doi: 10.1016/j.jcma.2017.12.005

Conflict of Interest: The authors declare that the research was conducted in the absence of any commercial or financial relationships that could be construed as a potential conflict of interest.

Copyright $\odot 2020 \mathrm{He}, \mathrm{He}, \mathrm{Hou}$, Chen, Huang, Yang, Luo and Li. This is an openaccess article distributed under the terms of the Creative Commons Attribution License (CC BY). The use, distribution or reproduction in other forums is permitted, provided the original author(s) and the copyright owner(s) are credited and that the original publication in this journal is cited, in accordance with accepted academic practice. No use, distribution or reproduction is permitted which does not comply with these terms. 